\title{
Neutrino Physics
}

\author{
Wick C. Haxton \\ Institute for Nuclear Theory, Box 351550, \\ and Department of Physics, Box 351560, \\ University of Washington, Seattle, WA 98195 \\ and \\ Barry R. Holstein \\ Institut für Kernphysik \\ Forschungszentrum Jülich \\ D-52425 Jülich, Germany \\ and \\ Department of Physics and Astronomy \\ University of Massachusetts, Amherst, MA 01003 \\ February 1, 2008
}

\begin{abstract}
The basic concepts of neutrino physics are presented at a level appropriate for integration into elementary courses on quantum mechanics and/or modern physics.
\end{abstract}




\section{Introduction}

The neutrino has been in the news recently, with reports that the $\mathrm{Su}$ perKamiokande collaboration - which operates a 50,000 ton detector of ultrapure water isolated deep within the Japanese mine Kamiokande - has found evidence of a nonzero neutrino mass [1]. The neutrino, a ghostly particle which can easily pass through the entire earth without interacting, has long fascinated both the professional physicist and the layman, as this ditty from writer John Updike [2] attests

Neutrinos, they are very small

They have no charge and have no mass

And do not interact at all.

The earth is just a silly ball

To them, through which they simply pass,

Like dustmaids down a drafty hall

Or photons through a sheet of glass.

They snub the most exquisite gas,

Ignore the most substantial wall,

Cold-shoulder steel and sounding brass,

Insult the stallion in his stall,

And, scorning barriers of class,

Infiltrate you and me! Like tall

And painless guillotines, they fall

Down through our heads into the grass.

At night, they enter at Nepal

And pierce the lover and his lass

From underneath the bed-you call

It wonderful; I call it crass.

We present this pedagogical discussion of basic neutrino physics in the hope that aspects of this topical and fascinating subject can be integrated into introductory courses, providing a timely link between classroom physics and science news in the popular press. In this way an instructor may be able to build on student curiosity in order to enrich the curriculum with some unusual new physics. In this spirit we present below some of the basic physics 
underlying massive neutrinos and neutrino mixing, as well as other properties of neutrinos relevant to both terrestrial experiments and astrophysics.

\section{Neutrinos: History}

We begin with a bit of history - an interesting and more detailed discussion can be found in Laurie Brown's article in the September 1978 issue of Physics Today [3]. Nuclear beta decay is a natural form of radioactivity wherein a parent nucleus decays to a daughter with the same atomic mass, but an atomic number changed by one unit, with the missing charge carried off by an electron or positron

$$
(A, Z) \rightarrow(A, Z \pm 1)+e^{\mp}
$$

This is quite literally nuclear transmutation of the type that fascinated alchemists of an earlier age. One well-known example is the decay of a free neutron into a proton and electron, with a half life of about 10 minutes. Another is the decay of a bound neutron in tritium to produce an electron and ${ }^{3} \mathrm{He}$ with a half life of 12.26 years: the effects of the nuclear binding in changing the energy released in the decay is responsible for the great increase in the half life. At the end of the 1920's the existence of such beta emitters was well established. However, the spectrum of the emitted electrons was puzzling. If beta decay occurs from rest into a two-body final state as given in Eq. 1, momentum conservation would require the momenta of the emitted electron and recoiling nucleus to be equal and opposite. Energy conservation would then fix the outgoing electron energy which, because the nucleus is heavy and thus recoils with a negligible velocity, is nearly equal to the difference of the parent and daughter nuclear masses (known as the reaction energy release or Q-value)

$$
Q \simeq M(A, Z)-M(A, Z \pm 1)
$$

As the Q-value in the beta decay of tritium is $18.6 \mathrm{keV}$, one would expect a monochromatic spectrum with all emitted electrons having this energy. Instead experimentalists found a continous spectrum of electron energies ranging from the rest mass $m_{e}$ to the Q-value, peaking at an energy about halfway in between, as shown in Figure 1. Various explanations were considered-

Niels Bohr even proposed the possibility that energy conservation was no 


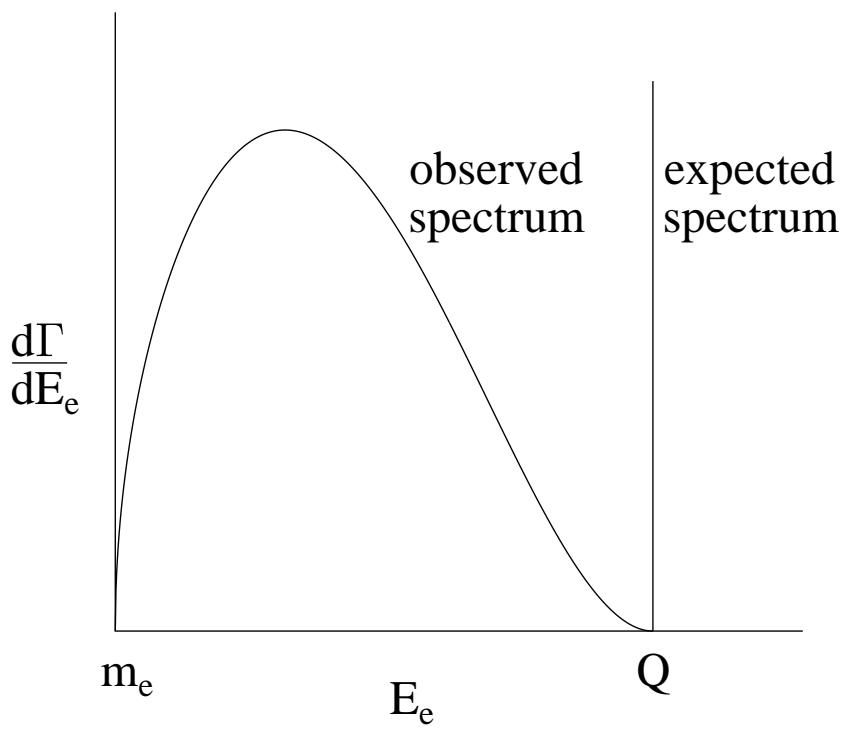

Figure 1: The $\beta$ energy spectrum for decay into a heavy daughter nucleus, electron, and neutrino is compared to the monoenergetic spectrum for decay into a daughter nucleus and electron, only. The spectrum is idealized: distortions due to the Coulomb interaction between the electron and daughter nucleus have been neglected.

longer exact in such subatomic processes, and rather preserved only in a statistical sense, somewhat in analogy with the second law of thermodynamics! However, in a letter dated December, 1930, Pauli suggested an alternative explanation - that an unobserved light neutral particle (called by him the "neutron" or neutral one but later renamed by Fermi the "neutrino" or little neutral one) accompanied the outgoing electron and carried off the missing energy that was required to satisfy energy conservation. Pauli offered this explanation tentatively as a "desperate remedy" to solve the energy problem. Although he publicized it in various talks over the next three years, no publication occurred until his contribution to the Seventh Solvay Conference in October 1933 [4]. He also proposed (correctly) that the neutrino was a particle carrying spin $1 / 2$ in order to satisfy angular momentum conservation and statistics. 
Fermi was present at a number of Pauli's presentations and discussed the neutrino with him on these occasions. In 1934 he published his insightful model for the beta decay process, and indeed for weak interactions in general [5]. He described beta decay in analogy with Dirac's successful model of the electromagnetic interaction, wherein two charged particles interact via the exchange of a (virtual) photon that is produced and then absorbed by the electromagnetic currents associated with the particles ( $c f$. Figure 2a). Fermi represented the weak interaction in terms of the product of weak "currents," one connecting the initial and final nucleon and the other connecting the final state electron/positron and Pauli's neutrino ( $c f$. Figure $2 \mathrm{~b}$ ). In electromagnetism the virtual photon connects the two currents at distinct points in space-time: indeed the masslessness of the photon is the reason for the long-range Coulomb force. In his weak interaction theory, however, Fermi connected the currents at the same space-time point, in effect assuming that the weak interaction is very short ranged. The strength of the interaction was determined by an overall coupling strength $G_{F}$

$$
\mathcal{H}_{w}=\frac{G_{F}}{\sqrt{2}} \psi_{p}^{\dagger} j_{\mu} \psi_{n} \psi_{e}^{\dagger} j^{\mu} \psi_{\nu}
$$

As written above, one of the weak currents is associated with the conversion of a neutron into a proton, and the other with the production out of the vacuum of an electron and antineutrino, which carry off almost all of the released energy. The electron spectrum predicted by this weak Hamiltonian can be readily calculated by using Fermi's golden rule for the differential decay rate, yielding in the no-nuclear-recoil approximation

$$
\begin{aligned}
d \Gamma & \sim\left(\frac{G_{F}}{\sqrt{2}}\right)^{2} \frac{d^{3} p_{e}}{(2 \pi)^{3}} \frac{d^{3} p_{\nu}}{(2 \pi)^{3}} 2 \pi \delta\left(Q-E_{e}-E_{\nu}\right)\left|M_{w}\right|^{2} \\
& \sim\left(\frac{G_{F}}{\sqrt{2}}\right)^{2} \frac{(4 \pi)^{2}}{(2 \pi)^{5}} d E_{e} E_{e} p_{e} \sqrt{\left(Q-E_{e}\right)^{2}-m_{\nu}^{2}}\left(Q-E_{e}\right)\left|M_{w}\right|^{2}
\end{aligned}
$$

where $M_{w}$ is the nuclear matrix element. Taking $m_{\nu}=0$ and assuming $\left|M_{w}\right|^{2} \sim$ constant we find

$$
\frac{d \Gamma}{d E_{e}} \sim p_{e} E_{e}\left(Q-E_{e}\right)^{2}
$$

where $p_{e}$ and $E_{e}$ are the momentum and energy of the electron. The excellent fit to experimentally measured spectra was an important confirmation of 


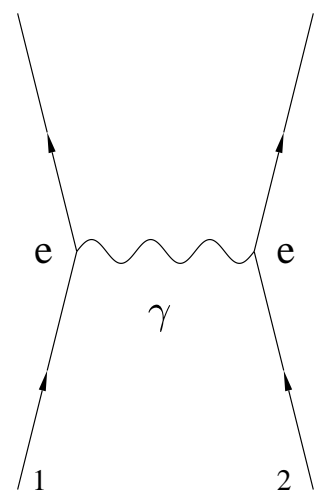

a)

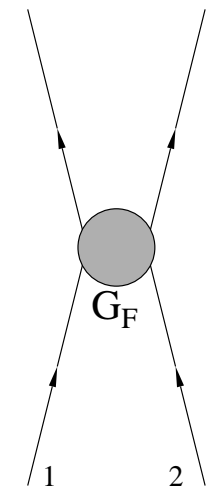

b)

Figure 2: Simple diagrammatic representation of a) the electromagnetic interaction arising from the exchange of a virtual photon between the electromagnetic currents generated by two charge particles and b) the weak interaction as a local product of two weak currents.

Fermi's theory, and thus of Pauli's postulate of the neutrino. Yet it would take another two decades to detect this elusive particle directly.

The reason behind the difficulty of direct detection of the neutrino can be seen in the size of the weak coupling $G_{F}$. From the 887 second lifetime of the neutron one finds that $\Gamma_{n}=\hbar / \tau_{n} \sim 7 \times 10^{-28} \mathrm{GeV}$, while Fermi's theory gives

$$
\begin{aligned}
\Gamma_{n} & \sim\left|\frac{G_{F}}{\sqrt{2}}\right|^{2} \int \frac{d^{3} p_{e}}{(2 \pi)^{3}} \frac{d^{3} p_{\nu}}{(2 \pi)^{3}} 2 \pi \delta\left(m_{n}-m_{p}-E_{e}-E_{\nu}\right)\left|M_{w}\right|^{2} \\
& =\frac{G_{F}^{2}}{2} \frac{(4 \pi)^{2}}{(2 \pi)^{5}} \int_{m_{e}}^{m_{n}-m_{p}} d E_{e} E_{e} p_{e}\left(m_{n}-m_{p}-E_{e}\right)^{2}\left|M_{w}\right|^{2} \\
& \simeq 4.59 \times 10^{-19} \mathrm{GeV}^{5} G_{F}^{2}\left|M_{w}\right|^{2}
\end{aligned}
$$

As $\left|M_{w}\right|^{2} \sim 6$, one finds $G_{F} \sim 10^{-5} \mathrm{GeV}^{-2}$. In order to understand why this interaction is called "weak", we note that the contemporary picture is that $G_{F}$ can be understood in analogy with the electromagnetic interaction as the result of an exchange of a virtual but very massive and charged "photon"the W-boson - so that $G_{F} \sim e^{2} / M_{W}^{2}$ with $M_{W} \sim 80 \mathrm{GeV}$. By the uncertainty 
principle, however, a virtual particle with such a heavy mass can propagate a distance $\Delta x \sim c \Delta t \sim \hbar c / M_{W} \sim 0.002 \mathrm{fm}$., i.e. a very small fraction of the nucleon radius. It is this exceeding small interaction range - which allows point particles, such as the neutrino and the quarks that are the underlying consituents of a nucleon, to interact only if they fortuitously pass very close to one another - that is responsible for the weakness of the weak interaction.

Fermi's theory is a relativistic quantum field theory, wherein a given field operator $\psi_{q}$ represents both an annihilation operator for generic particle $q$ as well as a creation operator for the corresponding antiparticle $\bar{q}$. Thus Eq. 3 (and its generalization for beta decay of protons within the nucleus) contains not only the interaction for beta decay- $n \rightarrow p e^{-} \bar{\nu}$ and $p \rightarrow n e^{+} \nu$ - but also

i) electron capture, where an atomic electron orbiting the nucleus interacts with one of the nuclear protons, converting it to a neutron and producing an outgoing neutrino, $e^{-}+p \rightarrow n+\nu$. (An analogous process for positrons, $e^{+}+n \rightarrow p+\bar{\nu}$, is important in the hot plasmas encountered in the big bang and in explosive stellar environments.)

ii) The charged current neutrino reactions $\nu+n \rightarrow p+e^{-}$and $\bar{\nu}+p \rightarrow n+e^{+}$ (which are the inverses of the reactions in i), but are often referred to as "inverse beta decay").

iii) The exotic resonant reactions $\bar{\nu}+e^{-}+p \rightarrow n$ and $\nu+e^{+}+n \rightarrow p$, the true inverse reactions of beta decay. The first can occur in an atom; both can take place in astrophysical plasmas.

Reaction ii) is the one relevant for neutrino detection, as the produced electron/positron and nuclear transformation are signals for a neutrino interaction. Such a process is characterized by the scattering cross section $\sigma$, which when multiplied by the incident particle flux and the number of scattering targets and integrated over solid angles, yields the number of scattering events per unit time. The cross section has the dimensions of area. In the approximation that the neutron is much heavier than the positron

$$
\sigma \sim\left|\frac{G_{F}}{\sqrt{2}}\right|^{2} \int \frac{d^{3} p_{e}}{(2 \pi)^{3}} 2 \pi \delta\left(m_{p}+E_{\nu}-m_{n}-E_{e}\right)\left|M_{w}\right|^{2} \sim \frac{G_{F}^{2}}{2 \pi} p_{e} E_{e}\left|M_{w}\right|^{2}
$$

For incident neutrino energies large compared to the electron rest mass this becomes $\sigma \sim G_{F}^{2} E_{\nu}^{2}\left|M_{w}\right|^{2} / 2 \pi \sim 10^{-44} \mathrm{~cm}^{2}$ for $E_{\nu} \sim 1 \mathrm{MeV}$. If we consider a 
single neutrino passing through a slab of material having a target density $\rho$ (e.g., $\sim 10^{23}$ atoms $/ \mathrm{cm}^{3}$ for typical materials), it would travel a distance

$$
\Delta x \sim 1 /(\rho \sigma) \sim 10^{21} \mathrm{~cm}
$$

before interacting, a distance equivalent to 100 billion earth radii! This is indeed a weak interaction! The only way to circumvent this problem is to have lots of low energy neutrinos. The orginal plan of a Los Alamos team led by Fred Reines and Clyde Cowan was to use a fission bomb to produce the needed neutrinos. Later, however, they decided to use a nuclear reactor, which produces large numbers of antineutrinos. Working at the Savannah River reactor, in South Carolina, which has a neutrino flux of about $10^{13}$ per square centimeter per second, they designed a detector consisting of two plastic tanks each filled with 200 liters of water, in which was dissolved cadmium chloride. The protons in the water provided the target for the reaction $\bar{\nu}+p \rightarrow n+e^{+}$, while the cadmium has a large cross section for neutron capture. The tanks were sandwiched between three scintillation detectors. The group looked for a signal consisting of gamma rays from the annihilation of the emitted positron on an electron followed closely (within a few microseconds) by gammas from the deexcitation of the cadium nucleus that had captured the neutron. The observed signal was correlated with the reactor being in operation. With this evidence they announced in 1956 that the neutrino had been detected [6], almost 25 years after Pauli's original suggestion. In 1996 Reines was awarded the Nobel Prize for this discovery. (Clyde Cowan had died years earlier.)

However, this was not the end of the story, but only the beginning. Indeed within seven years there was another Nobel-Prize-winning neutrino discovery: Lederman, Schwartz, and Steinberger demonstrated that there was more than one type of neutrino [7]. In order to explain their discovery we first provide a bit of theoretical background. As discussed above, the processes associated with Fermi's picture of beta decay are called "weak," characterized by rates or cross sections nearly twenty orders of magnitude smaller than those involving strongly interacting particles, such as the cross sections for scattering one nucleon off another. Particles, such as neutrinos, that do not participate in strong interactions are called "leptons." Thus the electron is also a lepton. (Of course, as the electron carries a charge, it has both electromagnetic and weak interactions, while we believe neutrinos react only weakly.) In the 1930's another charged particle was found which does not 
interact strongly - the muon. Except for the fact that it is about 200 times heavier, the muon's behavior is remarkably similar to that of the electron [ 8$]$. The muon, however, is unstable and decays in $\sim 10^{-6}$ sec into an electron and two neutral unseen particles that we now know are a neutrino and an antineutrino. This timescale is appropriate for a weak interaction, as can be seen from the estimate

$$
\begin{aligned}
\Gamma_{\mu} & =\frac{1}{\tau_{\mu}} \sim\left|\frac{G_{F}}{\sqrt{2}}\right|^{2} \int \frac{d^{3} p_{e}}{(2 \pi)^{3}} \frac{d^{3} p_{\nu}}{(2 \pi)^{3}} \frac{d^{3} p_{\bar{\nu}}}{(2 \pi)^{3}}(2 \pi)^{4} \delta^{4}\left(p_{\mu}-p_{e}-p_{\nu}-p_{\bar{\nu}}\right) \\
& \sim \frac{G_{F}^{2} m_{\mu}^{5}}{1636 \pi^{3}} \quad \text { i.e. } \quad \tau_{\mu} \sim 10^{-5} \mathrm{sec}
\end{aligned}
$$

Muon decay fits easily into Fermi's interaction provided the lepton current is generalized to

$$
J_{\mu}^{\mathrm{lep}}=\psi_{e}^{\dagger} j_{\mu} \psi_{\nu}+\psi_{\mu}^{\dagger} j_{\mu} \psi_{\nu}
$$

Then the product of the lepton current with its hermitian conjugate yields an interaction responsible for muon decay.

In order to understand the experiment of ref. [7], it is useful to go one step further and introduce modern quark notation. In the quark model the neutron is a composite object comprised of a pair of d quarks and a single $u$ quark, while the proton consists of a pair of u quarks and a single d quark. The weak current connecting the proton and neutron can then be replaced by a corresponding current connecting a u quark with a d quark,

$$
J_{\mu}^{\mathrm{had}}=\psi_{n}^{\dagger} j_{\mu} \psi_{p} \rightarrow \psi_{d}^{\dagger} j_{\mu} \psi_{u}
$$

As the field operator $\psi_{d}^{\dagger}$ can both create a $d$ quark and destroy the corresponding antiparticle $(\bar{d})$, this same current describes the quark component of the process where a $\bar{d} u$ system (i.e. a $\pi^{+}$meson) decays to a muon and a neutrino

$$
\pi^{+} \rightarrow \mu^{+}+\nu
$$

which is the dominant decay mode of the charged pion [9]. The experimenters of ref. [7] collided neutrinos from such decays with neutrons in an attempt to produce electrons and muons, as predicted by the current of Eq. 10. But they found only muons, not electrons. The explanation for this result is that neutrinos come in two distinct species, an electron type $\nu_{e}$ and a muon type 
$\nu_{\mu}$, with the weak current coupling electrons only to $\nu_{e}$ and muons only to $\nu_{\mu}$

$$
J_{\mu}^{\text {lep }}=\psi_{e}^{\dagger} j_{\mu} \psi_{\nu_{e}}+\psi_{\mu}^{\dagger} j_{\mu} \psi_{\nu_{\mu}}
$$

The neutrino produced in pion decay thus must be a $\nu_{\mu}$ and of the wrong type, or "flavor," to produce an electron. In 1977 a third charged lepton, the $\tau$, was discovered and another term has now been added to this equationthe coupling of the $\tau$ to its neutrino, the $\nu_{\tau}$. Measurements of the decay width of the neutral $Z$-boson [10 and astrophysical arguments based on the helium abundance in the universe [11] suggest that this may exhaust the set of lepton-neutrino pairs: there appear to be no more light neutrinos beyond the $\nu_{\tau}$.

The modern picture of the weak interaction consists not only of three doublets of charged lepton-neutrino pairs but also of three doublets (often called "generations") of charge $2 / 3$, charge $-1 / 3$ quarks - $(\mathrm{u}, \mathrm{d}),(\mathrm{c}, \mathrm{s}),(\mathrm{t}, \mathrm{b})$. The charged weak current then can be written as the sum of six separate currents connecting such quark and lepton doublets

$$
J_{\mu}=J_{\mu}^{\mathrm{had}}+J_{\mu}^{\mathrm{lep}}=\left(\psi_{d}^{\dagger} \psi_{s}^{\dagger} \psi_{b}^{\dagger}\right) U_{K M} j_{\mu}\left(\begin{array}{c}
\psi_{u} \\
\psi_{c} \\
\psi_{t}
\end{array}\right)+\left(\psi_{e}^{\dagger} \psi_{\mu}^{\dagger} \psi_{\tau}^{\dagger}\right) j_{\mu}\left(\begin{array}{c}
\psi_{\nu_{e}} \\
\psi_{\nu_{\mu}} \\
\psi_{\nu_{\tau}}
\end{array}\right)
$$

Low-energy weak interactions are then described by a effective currentcurrent interaction with a single overall coupling $G_{F}$

$$
\mathcal{H}_{w}=\frac{G_{F}}{\sqrt{2}} J_{\mu}^{\dagger} J^{\mu}
$$

Such a contact interaction is a good approximation at low energies to a more complete theory described in terms of the exchange of a heavy charged Wboson, as we mentioned earlier. Here $U_{K M}$ is a general unitary $3 \times 3$ matrix, which is not needed in the case of the lepton current due to the assumption in the standard model that the three neutrinos are degenerate. Consequently Fermi's weak interaction, in its modern guise, contains an enormous range of physical processes. In addition in 1972 a different kind of weak interaction was found, wherein a neutral current (which is diagonal in quark and lepton 
identities) is coupled to its hermitian conjugate.

$$
\begin{aligned}
& \mathcal{J}_{\mu}=\mathcal{J}_{\mu}^{\mathrm{had}}+\mathcal{J}_{\mu}^{\mathrm{lep}}=\left(\psi_{e}^{\dagger} \psi_{\mu}^{\dagger} \psi_{\tau}^{\dagger}\right) j_{\mu}^{\prime}\left(\begin{array}{c}
\psi_{e} \\
\psi_{\mu} \\
\psi_{\tau}
\end{array}\right)+\left(\begin{array}{lll}
\psi_{\nu_{e}}^{\dagger} & \psi_{\nu_{\mu}}^{\dagger} & \psi_{\nu_{\tau}}^{\dagger}
\end{array}\right) j_{\mu}^{\prime}\left(\begin{array}{c}
\psi_{\nu_{e}} \\
\psi_{\nu_{\mu}} \\
\psi_{\nu_{\tau}}
\end{array}\right) \\
& +\left(\psi_{u}^{\dagger} \psi_{c}^{\dagger} \psi_{t}^{\dagger}\right) j_{\mu}^{\prime}\left(\begin{array}{c}
\psi_{u} \\
\psi_{c} \\
\psi_{t}
\end{array}\right)+\left(\psi_{d}^{\dagger} \psi_{s}^{\dagger} \psi_{b}^{\dagger}\right) j_{\mu}^{\prime}\left(\begin{array}{c}
\psi_{d} \\
\psi_{s} \\
\psi_{b}
\end{array}\right)
\end{aligned}
$$

with

$$
\mathcal{H}_{w} \sim \frac{G_{F}}{\sqrt{2}} \mathcal{J}_{\mu}^{\dagger} \mathcal{J}^{\mu}
$$

In this case the interaction arises from the exchange of a heavy neutral particle - the Z-boson with mass $m_{Z} \sim 91 \mathrm{GeV}$ - and can again be taken to be of contact form for low-energy reactions.

Before leaving this historical journey it is useful to remark on one additional feature of the weak interaction important to modern studies - the handedness. As both quarks and leptons are spin-1/2 objects, they can be described by four-component Dirac fields and their currents can be expressed as bilinear forms of such fields connected by $4 \times 4$ Dirac matrices. Experimentally one finds that the proper combination is an equal mixture of polar and axial vector structures

$$
\psi_{a}^{\dagger} j_{\mu} \psi_{b} \equiv \psi_{a}^{\dagger} \gamma_{0} \gamma_{\mu}\left(1-\gamma_{5}\right) \psi_{b}
$$

The Dirac matrix

$$
1-\gamma_{5}=\left(\begin{array}{cc}
1 & -1 \\
-1 & 1
\end{array}\right)
$$

is called a "chirality" operator and, for particles of zero mass, projects out only "left-handed" particles - i.e., those whose spins are aligned opposite to their momenta. In order to see this, consider the Dirac equation for a free particle of mass $m$

$$
(i \not \partial-m) \psi(x)=0
$$

where $\not \partial=\partial_{0} \gamma_{0}-\vec{\partial} \cdot \vec{\gamma}$. We use the standard representation for the $4 \times 4$ Dirac matrices 12

$$
\gamma_{0}=\left(\begin{array}{cc}
1 & 0 \\
0 & -1
\end{array}\right) \quad \vec{\gamma}=\left(\begin{array}{cc}
0 & \vec{\sigma} \\
-\vec{\sigma} & 0
\end{array}\right)
$$


The positive energy plane wave solutions of Eq. 21 are well-known

$$
\psi(x)=\sqrt{\frac{E+m}{2 E}}\left(\begin{array}{c}
\chi \\
\frac{\vec{\sigma} \cdot \vec{p}}{E+m} \chi
\end{array}\right) \exp (-i p \cdot x)
$$

where $\chi$ is a two-component Pauli spinor. Then in the limit as $m \rightarrow 0$ and $\mathrm{E} \rightarrow|\vec{p}|$ this becomes

$$
\psi(x) \underset{m \rightarrow 0}{\longrightarrow} \psi_{0}(x)=\frac{1}{\sqrt{2}}\left(\begin{array}{c}
\chi \\
\vec{\sigma} \cdot \hat{p} \chi
\end{array}\right) \exp (-i p \cdot x)
$$

so that

$$
\left(1-\gamma_{5}\right) \psi_{0}(x)=\left\{\begin{array}{cc}
\psi_{0}(x) & \vec{\sigma} \cdot \hat{p} \chi=-\chi \\
0 & \vec{\sigma} \cdot \hat{p} \chi=\chi
\end{array}\right.
$$

as claimed. This result is important, as will be discussed in the next section, because the neutrino is either massless or extremely light. Therefore, since the neutrino interacts only via the weak interactions which involve the chirality operator $1-\gamma_{5}$, all neutrinos must be left-handed! [13] Similarly, it is easy to see that all antineutrinos must be right-handed.

Another way of stating this result is to say that, although Dirac spinors are four-component objects, those describing zero mass neutrinos involve only two of the four components. This "two-component neutrino" theory has been tested in a direct measurement of neutrino helicity in the reaction 14

$$
{ }^{152} \mathrm{Eu}\left(0^{-}\right)+e^{-} \rightarrow{ }^{152} \mathrm{Sm}^{*}\left(1^{-}\right)+\nu_{e} \rightarrow{ }^{152} \mathrm{Sm}\left(0^{+}\right)+\gamma+\nu_{e}
$$

The clever idea behind this scheme is that one can select those $\gamma$ 's from the decay of the Sm excited state which travel oppositely to the direction of the electron-capture $\nu_{e}$ 's (i.e. in the direction of the nuclear recoil) by having them resonantly scatter from a Sm target. By angular momentum conservation the helicity of the downward-going $\gamma$ is the same as that of the upward-traveling $\nu_{e}$. The results of the experiment strongly confirmed the two-component hypothesis.

It is interesting to note that the chirality structure of the weak current also explains why the decay of the charged pion proceeds predominantly via $\pi^{+} \rightarrow \mu^{+}+\nu_{\mu}$ rather than by the mode $\pi^{+} \rightarrow e^{+}+\nu_{e}$ which is strongly favored by phase space. The point is that if the positron were massless, 


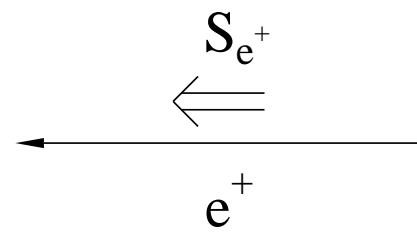

right-handed

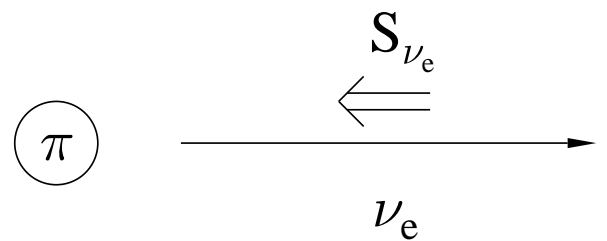

left-handed

Figure 3: Schematic representation of a pion at rest decaying to a massless positron and a neutrino. Such a decay is forbidden by angular momentum conservation.

it too would be described by a two-component theory and any such particle coupled to the weak interaction would have to be purely right-handed. Then, as diagrammed in Figure 3, the decay of a pion into a positron and a neutrino must be forbidden because angular momentum conservation prohibits the coupling of a right-handed positron and left-handed neutrino to a spinless system. Of course, in the real world the positron is light but not massless. Thus the positron decay of the charged pion is not completely forbidden but rather highly suppressed compared to its muonic counterpart-

$$
R_{e} \equiv \frac{\Gamma\left(\pi^{+} \rightarrow e^{+}+\nu_{e}\right)}{\Gamma\left(\pi^{+} \rightarrow \mu^{+}+\nu_{\mu}\right)}=\left(\frac{m_{e}}{m_{\mu}}\right)^{2}\left(\frac{m_{\pi}^{2}-m_{e}^{2}}{m_{\pi}^{2}-m_{\mu}^{2}}\right)^{2}=1.23 \times 10^{-4}
$$

which is confirmed by experiment[15]

$$
R_{e}^{e x p}=(1.230 \pm 0.004) \times 10^{-4}
$$

It is interesting to note one final point about neutrinos which has a close connection to helicity. The reader will have noticed that we have throughout distinguished the $\nu_{e}$ produced when a proton beta decays in a nucleus from the $\bar{\nu}_{e}$ produced in neutron beta decay. The concept of a distinct antiparticle is certainly clear for charged leptons like the electron, as its antiparticle - the positron-carries the opposite charge. More generally, particle-antiparticle conjugation reverses the signs of all of a particle's additively conserved quantum numbers. The neutrino is immediately seen to be quite interesting then, as it lacks a charge, magnetic moment, or other measured quantum number 
that would necessarily reverse under such an operation - it is unique among the leptons and quarks in that the existence of a distinct antiparticle is an open question.

Early on, before the handedness of the weak interaction was discovered, there appeared to be a simple test of the particle-antiparticle properties of the neutrino. If one defines the $\nu_{e}$ as the neutrino produced when a proton decays in a $\beta^{+}$source, then one finds that $\nu_{e}$ 's produce electrons by the reaction

$$
\nu_{e}+n \rightarrow p+e^{-}
$$

but not positrons in the analogous reaction

$$
\nu_{e}+p \nrightarrow n+e^{+}
$$

Similarly if we define the $\bar{\nu}_{e}$ as the particle produced in the $\beta^{-}$decay of the neutron decay, then $\bar{\nu}_{e}$ 's produce positrons by the reaction

$$
\bar{\nu}_{e}+p \rightarrow n+e^{+}
$$

but not electrons by the reaction

$$
\bar{\nu}_{e}+n \not p+e^{-}
$$

Thus it would appear that the $\nu_{e}$ and the $\bar{\nu}_{e}$ are operationally distinct. In fact, the absence of the reactions in Eqs. 29,31 became apparent around 1950 from an experiment done by nature, a form of natural radioactivity known as double beta decay. If, for example, the reaction in Eq. 31 were allowed, certain nuclei could undergo the second-order weak decay

$$
(A, Z) \rightarrow(A, Z+1)+e^{-}+\bar{\nu}_{e} \rightarrow(A, Z+2)+2 e^{-}
$$

where the neutrino produced in the first decay is reabsorbed by the nucleus, producing a final state with two electrons and no neutrinos. The absence of such "neutrinoless double beta decay," which has a distinctive experimental signal because the entire energy release is carried off by the electrons, thus seemed to show that the $\nu_{e}$ and $\bar{\nu}_{e}$ were indeed distinct particles [16]. This prompted the introduction of a distinguishing quantum number, lepton number. The $\nu_{e}$ and electron were assigned $l_{e}=+1$, the $\bar{\nu}_{e}$ and positron $l_{e}=-1$. The assumption of an additively conserved lepton number in weak 
interactions then allows the reactions in Eqs. 28,30, but explains the absence of the reactions in Eqs. 29, 31, 32. A neutrino with a distinct antineutrino is called a Dirac neutrino.

However the discovery of the apparent exact handedness of the weak interaction invalidates this simple conclusion. All of the results are also explained by the assignments

$$
\nu_{e} \rightarrow \nu_{e}^{L H} \text { and } \quad \bar{\nu}_{\mathrm{e}} \rightarrow \nu_{\mathrm{e}}^{\mathrm{RH}}
$$

and a weak interaction that violates parity maximally. Here $R H$ denotes a right-handed particle and $L H$ a left-handed one. Thus the possibility that the neutrino is its own antiparticle - a so-called Majorana neutrino - is still open. In this case a reaction like that of Eq. 32 is not forbidden by an exact additive conservation law, but rather by helicity. Therefore if a Majorana neutrino had a small mass, neutrinoless double beta decay would occur, but the decay rate would by suppressed by the small quantity

$$
\left(\frac{m_{\nu}}{E_{\nu}}\right)^{2}
$$

where $E_{\nu} \sim 50 \mathrm{MeV}$ is an energy characteristic of the virtual neutrino emitted and reabsorbed in the decay. Modern searches for neutrinoless double beta have established limits on half lives of $\sim 10^{25} \mathrm{y}$, corresponding to a Majorana neutrino mass below $1 \mathrm{eV}$ [17].

Given that the familiar charged leptons have only Dirac masses, it is natural to ask why neutrinos, which can have two kinds of masses, would then be the only massless leptons in the standard model. The absence of Dirac neutrino masses in the standard model follows from the need to have both left-hand and right-handed fields in order to construct such masses. We have noted that neutrinos interact only weakly and that weak interactions involve only left-handed components of the fields. The standard model, being very economical, has no right-handed neutrino fields and thus no Dirac neutrino masses. However, the absence of Majorana masses has a more subtle explanation. One can construct a left-handed Majorana mass with the available standard model neutrino fields, but it turns out this term is not "renormalizable," i.e., it generates infinities in the theory. Our point-like Fermi $\beta$ decay theory is another example of a nonrenormalizable theory, though it works quite well in the domain of low-energy weak interactions. If we were 
to relegate the standard electroweak model to a similar status - that of an effective theory - Majorana mass terms could then be introduced. In effect, most extensions of the standard model do precisely that, and also generally introduce new fields such as those creating right-handed neutrinos. Thus almost all theorists, believing the standard model is incomplete and must be extended in some such ways, also believe that neutrinos have masses. Indeed, the puzzle is rather to explain why these masses are so much smaller than those of charged particles.

With this historical background out of the way, we now move to consider aspects of the neutrino that have recently been in the news - masses and mixings.

\section{Neutrino Mass: Direct Measurements}

The issue of whether the neutrino has a nonzero mass has been long one of interest. That any such mass must be small could be seen from the feature that the maximum energy measured in the beta spectrum agreed to high precision with the mass difference of initial and final nuclear states. However, Fermi, in his seminal paper on beta decay, noted that this question could be answered more definitively by carefully studying the endpoint of the electron spectrum - it is possible to plot the spectrum in such a way that a nonzero mass would be revealed as a distortion at the endpoint tangent to (perpendicular to ) the energy axis, as shown in Figure 4. In the years since Fermi's paper there has been a series of such measurements, with steadily increasing precision. It is clear that use of a $\beta$ decay parent nucleus with a relatively low Q-value is helpful, as a larger fraction of total decays then resides within a given interval from the endpoint. Most experimenters have selected tritium, which has an $18.6 \mathrm{keV}$ endpoint. An early tritium measurement by Hamilton, Alford, and Gross found an upper limit $m\left(\bar{\nu}_{e}\right) \lesssim 250 \mathrm{keV}[18$. A few years later, Bergkvist, by combining electrostatic and magnetic spectrometric methods, was able to reduce the limit substantially $-m\left(\bar{\nu}_{e}\right) \lesssim 60 \mathrm{eV}$ [19]. Then in 1980 Lubimov et al., using a high-precision toroidal spectrometer and tritium in the form of the valine molecule $\left(\mathrm{C}_{5} \mathrm{H}_{11} \mathrm{NO}_{2}\right)$, claimed the first nonzero mass $-14 \mathrm{eV} \lesssim m\left(\bar{\nu}_{e}\right) \lesssim 46 \mathrm{eV}$, 20], a result that set off a flurry of new, high precision experiments. Before discussing the results, however, we first examine one of the reasons these experiments are important - the 


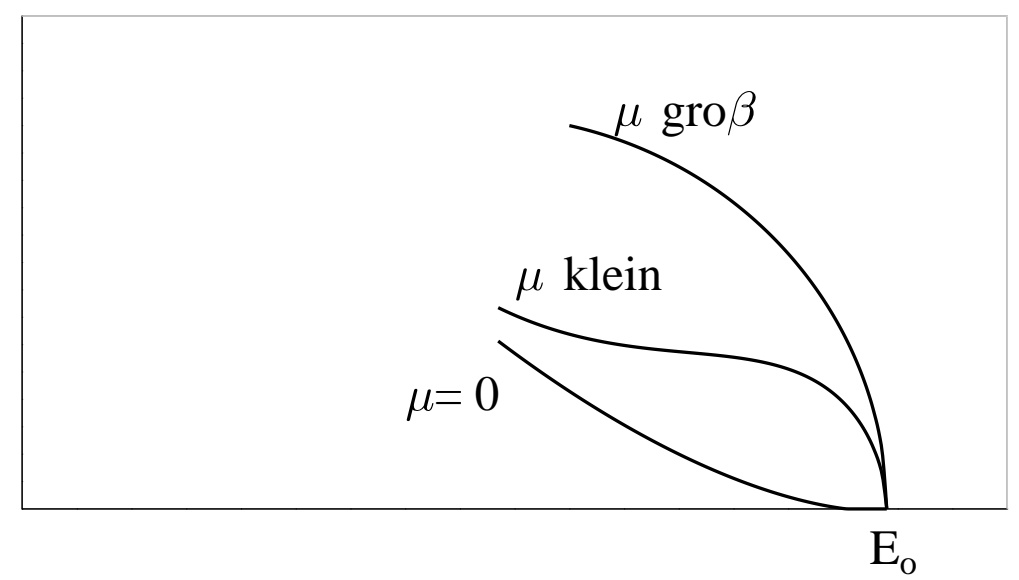

Figure 4: A reproduction of curves from Fermi's paper (Ref. [5]) showing the shape of the $\beta$ spectrum near the endpoint for the cases of zero and nonzero neutrino masses.

cosmological significance of a massive neutrino.

We are all aware that at the present time the universe is expanding. However, what will be its ultimate fate? Will it continue to expand forever, or will the expansion slow and finally reverse? In order to see what role neutrinos may play in answering this question, we explore their effects in an expanding homogeneous and isotropic universe. Consider a small test mass $m$ which sits on the surface of a spherical chunk of this universe having radius $\mathrm{R}$. If the mean energy density of the universe is $\rho$, then the mass contained inside the spherical volume is

$$
M(R)=\frac{4}{3} \pi R^{3} \rho
$$

The potential energy of the test mass, as seen by an observer at the center of the sphere, is

$$
U=-G \frac{M(R) m}{R}
$$

while its kinetic energy is

$$
T=\frac{1}{2} m v^{2}=\frac{1}{2} m\left(\frac{d R}{d t}\right)^{2}
$$


By Hubble's Law the expansion velocity is given by

$$
v=H R
$$

where $H=\frac{1}{R} \frac{d R}{d t}$ is the Hubble constant. Although the size of $H$ is still uncertain, most values are in the range

$$
50 \mathrm{~km} / \mathrm{sec} / \text { Megaparsec } \lesssim H_{\exp } \lesssim 100 \mathrm{~km} / \mathrm{sec} / \text { Megaparsec }
$$

(with perhaps $\sim 65 \mathrm{~km} / \mathrm{sec} /$ Megaparsec being the best value). The total energy of the test particle is then

$$
E_{\mathrm{tot}}=T+U=\frac{1}{2} m R^{2}\left(H^{2}-\frac{8}{3} \pi \rho G\right)
$$

and the fate of the universe depends on the sign of this number, or equivalently with the relation of the density to a critical value

$$
\rho_{c}=\frac{3 H^{2}}{8 \pi G} \sim 2 \times 10^{-29} \mathrm{~g} / \mathrm{cm}^{3},
$$

i.e.

$$
\begin{aligned}
& \rho<\rho_{c} \Rightarrow \text { continued expansion } \\
& \rho>\rho_{c} \Rightarrow \text { ultimate contraction }
\end{aligned}
$$

It is not yet clear which situation describes our universe. Analysis of the dynamics of gravitationally bound systems via the virial theorem and comparison with measured luminosities yields the "visible" mass density

$$
\rho_{\mathrm{vis}} \simeq 0.02 \rho_{c}
$$

However, Doppler studies of the rotation rates of spiral galaxies indicate that these systems are much more massive than their luminosities seem to suggest

$$
\rho_{\text {rot }} \sim 20 \rho_{\text {vis }}
$$

The origin of the "dark matter" responsible for this discrepancy is a matter of current study: there are several possibilities, including massive neutrinos. But regardless of the origin of the dark matter, it appears that the energy density of our universe is within an order of magnitude of the critical value required for closure. 
Before discussing the relevance of neutrinos to the dark matter, we consider the more familiar case of the relic photon spectrum, which has been carefully studied recently via the COBE satellite. In the early universe electromagnetic interactions such as

$$
e^{+}+e^{-} \leftrightarrow \gamma+\gamma, \quad \gamma+e^{-} \leftrightarrow \gamma+e^{-}
$$

kept the photons in thermal equilibrium with the electrons and nucleons. However once the universe cooled sufficiently - to $T \sim 1 \mathrm{eV}$ at about $10^{5}$ years after the big bang - electrons and protons combined to form neutral hydrogen. The absence of charged particles rendered the universe transparent to photons, which then thermally decoupled from the rest of the matter. At the time of "recombination" - the epoch when neutral hydrogen is formed the photons were characterized by a blackbody spectrum at a temperature $T_{\gamma}^{\text {recomb }} \sim 4000 \mathrm{~K}$. Because of the subsequent expansion of the universe, the spectrum today has been redshifted to a temperature $T_{\gamma}^{\text {now }} \sim 2.72 \mathrm{~K}$, as was established in the COBE measurements. The energy density of these relic photons is given by Stefan's Law

$$
\rho_{\gamma}=2 \int \frac{d^{3} q}{(2 \pi)^{3}} \frac{q}{\exp (q / T)-1}=4 \sigma T_{\gamma}^{4} \sim 4 \times 10^{-34} \mathrm{~g} / \mathrm{cm}^{3}
$$

where $\sigma=\pi^{2} / 60$ is the Stefan-Boltzmann constant.

An analogous relic neutrino spectrum must exist from the early universe. Such neutrinos were originally kept in thermal equilibrium with electrons and other matter by the weak interactions

$$
e^{+}+e^{-} \leftrightarrow \nu_{e}+\bar{\nu}_{e}, \quad e^{ \pm}+\nu_{e} \leftrightarrow e^{ \pm}+\nu_{e}, \quad e^{ \pm}+\bar{\nu}_{e} \leftrightarrow e^{ \pm}+\bar{\nu}_{e}
$$

However, once the temperature cooled to about $10^{10} \mathrm{~K}$ the weak reaction rates - which depend on $\sigma_{\text {weak }} n v$, where $n$ is the lepton density and $v$ the relative velocity, and vary as $T^{5}$ — can no longer keep up with the expansion rate, $H \sim \dot{R} / R \sim T^{2}$, which slows less rapidly. The neutrinos then drop out of equilibrium with the charged leptons. From this point they are essentially decoupled from the rest of the universe, but, of course, cool as expansion proceeds. The neutrino energy density at the present time is given by

$$
\rho_{\nu}=\frac{7}{2} N_{\nu} \sigma T_{\nu}^{4}
$$


where $N_{\nu}=3$ is the number of "massless" $\left(m_{\nu}<T\right)$ two-component neutrino generations 21]. Note, however, that the present neutrino and photon temperatures are different: while above $\sim 10^{10} \mathrm{~K}$ the reaction $e^{+}+e^{-} \leftrightarrow \gamma+\gamma$ proceeds in both directions, below this temperature the photon energy is no longer sufficient to produce pairs. Thus subsequent reactions proceed only to the right, resulting in a reheating process that raises the photon temperature with respect to the neutrinos by the factor

$$
\left(\frac{\rho_{\gamma}+\rho_{e^{-}}+\rho_{e^{+}}}{\rho_{\gamma}}\right)^{\frac{1}{3}}=\left(\frac{11}{4}\right)^{\frac{1}{3}}
$$

The present-day relic neutrino temperature is thus

$$
T_{\nu}=T_{\gamma}\left(\frac{4}{11}\right)^{\frac{1}{3}}=1.94 K
$$

The corresponding average neutrino energy is only $\sim 10^{-3} \mathrm{eV}$, so that scattering cross sections are unobservably small. But such relic neutrinos do contribute to the mass density of the universe, yielding in the case of massless neutrinos

$$
\rho_{\nu}=\rho_{\gamma} \times \frac{7}{8} \times N_{\nu} \times\left(\frac{4}{11}\right)^{\frac{4}{3}} \simeq 0.7 \rho_{\gamma}
$$

If, however, neutrinos have a nonzero mass, then a contribution comparable to the critical density results if the sum of the masses for the three neutrino species is as little as $\sim 25 \mathrm{eV}$. Thus neutrino masses easily compatible with existing experimental limits could close the universe and ultimately lead it to recollapse. In particular, since this number is within the range found in ref. 20] for the electron antineutrino alone, confirmation of that experiment was clearly crucial to cosmology.

Stimulated by the Lubimov result, several groups attempted improved versions of this experiment. One of the criticisms of the Russian experiment was the use a tritiated valine source, which introduced a substantial uncertainty because of binding effects and because the contribution of molecular excited states, populated in the beta decay, to the energy loss could not be calculated easily. Thus a group at Los Alamos used a much simpler source, gaseous tritium molecules, tackling at the same time the serious safety issues associated with handling a kilocurie of this gas. After a series of measurements with a carefully constructed magnetic spectrometer that filled an entire 
room, they found $m_{\bar{\nu}_{e}} \lesssim 9.3 \mathrm{eV}$ [22]. An experiment at Mainz using a frozen tritium source reported a similar limit, $m_{\bar{\nu}_{e}} \lesssim 7.2 \mathrm{eV}$ [23], and a Livermore group using gaseous tritium and a toroidal magnetic spectrometer achieved comparable statistics [24]. All of these experiments, however, were troubled by a puzzling excess of events near the endpoint. Although this problem has been described as a negative value for $m_{\bar{\nu}_{e}}^{2}(!)$, in fact this was too simple a characterization: each of the groups described the anomaly with a different functional form. While standard statistical techniques were then used to establish the above bounds on $m_{\bar{\nu}_{e}}^{2}$, it is apparent than an unknown systematic contributing excess events at the endpoint could also mask the effects of a positive $m_{\bar{\nu}_{e}}^{2}$. This has tended to weaken the community's confidence in the stated bounds. Ongoing experiments at Mainz and Troitsk now claim limits of $\sim(3-5) \mathrm{eV}$ and, while improved resolution and understanding of energy loss in the target has significantly mitigated the negative $m_{\bar{\nu}_{e}}^{2}$ problem, certain anomalies remain in the endpoint region.

Because of this situation, it was fortunate that a special event occurred that established an independent bound on the $\bar{\nu}_{e}$ mass. This was the observation of Supernova 1987A, which was found in the southern hemisphere on February 23rd of that year-cf. Figure 5. SN1987A resulted from the explosion of a star in the Large Magellanic Cloud about 170,000 years ago, the light (and neutrinos) from which finally reached earth in 1987. This was the first such optical supernova in our vicinity in nearly four hundred years, the previous occurrence having been noted by Kepler in 1604!

A brief discussion of stellar evolution is needed in order to make the connection between SN1987A and neutrinos. Small stars like our sun live relatively quiescent lives, spending billions of years slowly burning hydrogen to helium in their hot dense cores, with the liberated energy maintaining the electron gas pressure that stabilizes the star against gravitational collapse. A Type II supernova - the type to which SN1987A belongs - is the last evolutionary stage of a more massive star, in excess of 10 solar masses. Like our sun, such a star begins its lifetime burning the hydrogen in its core under the conditions of hydrostatic equilibrium. When the hydrogen is exhausted, the core contracts until the density and temperature are reached where helium can ignite via the $3 \alpha \rightarrow{ }^{12} \mathrm{C}$ reaction. The He is then burned to exhaustion. This pattern (fuel exhaustion, contraction, and ignition of the ashes of the previous burning cycle) repeats several times, leading finally to the explosive burning of ${ }^{28} \mathrm{Si}$ to Fe. For a heavy star, the evolution is rapid: the star 
Figure 5: Views of a region in the Large Magellanic Cloud before (right) and after (left) the morning of February 27, 1987: SN1987A is clearly visible. Copyright Anglo-Australian Observatory. Photograph by David Malin (http://www.aao.gov.au/local/www/dfm/aat050.htm]).

has to work harder to maintain itself against its own gravity, and therefore consumes its fuel faster. A 25 solar mass star would go through all of these cycles in about $7 \mathrm{My}$, with the final explosive Si burning stage taking only a few days!

Iron is the most strongly bound nucleus in the periodic table. Thus once the $\mathrm{Si}$ burns to produce Fe, there is no further source of nuclear energy with which to support the star. So, as the last remnants of nuclear burning take place, the core is largely supported by the electron degeneracy pressure. When enough ash accummulates so that the iron core exceeds the Chandrasekhar limit [25] - a limit of about 1.4 solar masses above which it is no longer stable-the core begins to collapse. Gravity does work on the infalling matter, leading to rapid heating and compression of the iron, and ultimately "boiling off" $\alpha$ 's and a few nucleons from the nuclei. At the same time, the electron chemical potential is increasing, making electron capture on nuclei and any free protons favorable,

$$
e^{-}+p \rightarrow \nu_{e}+n \text {. }
$$

Both the electron capture and the nuclear excitation and disassociation take energy out of the electron gas, which is the star's only source of support. 
This means that the collapse is very rapid. Indeed, numerical simulations find that the iron core of the star $(\sim 1.2-1.5$ solar masses $)$ collapses at about 0.6 of the free fall velocity.

In the early stages of the infall the $\nu_{e}$ 's readily escape. But neutrinos become trapped when a density of $\sim 10^{12} \mathrm{~g} / \mathrm{cm}^{3}$ is reached, at which point they begin to scatter off the matter through both charged current and coherent neutral current processes. The neutral current neutrino scattering off nuclei is particularly important, as the scattering cross section involves the total nuclear weak charge, which is approximately the neutron number. This process transfers very little energy because the mass energy of the nucleus is so much greater than the typical energy of the neutrinos. But momentum is exchanged. Thus the neutrino "random walks" out of the star, frequently changing directions. When the neutrino mean free path becomes sufficiently short, the "trapping time" of the neutrino begins to exceed the time scale for the collapse to be completed. This occurs at a density of about $10^{12} \mathrm{~g} / \mathrm{cm}^{3}$, or somewhat less than $1 \%$ of nuclear density. After this point, the energy released by further gravitational collapse is trapped within the star. If we take a neutron star of 1.4 solar masses and a radius of $10 \mathrm{~km}$, an estimate of this gravitational energy is

$$
\frac{G M^{2}}{2 R} \sim 2.5 \times 10^{53} \mathrm{ergs} .
$$

The collapse continues until nuclear densities are reached. As nuclear matter is rather incompressible (compression modulus $\sim 300 \mathrm{MeV}$ ), the nuclear equation of state then halts the collapse: maximum densities of 3-4 times nuclear density are reached, e.g., perhaps $6 \cdot 10^{14} \mathrm{~g} / \mathrm{cm}^{3}$. This sudden braking of the collapse generates a series of pressure waves which travel outward through the iron core and collect at the sonic point (where the infall velocity of the iron matches the sound speed in iron), eventually forming a shock wave that travels out through the mantle of the star. Theory suggests that this shock wave in combination neutrino heating of the matter is responsible for the ejection of the mantle - and thus the spectacular optical display we saw in 1987.

Yet this visible display represents less than $1 \%$ of the energy locked inside the protoneutron star. After the core collapse and shock wave formation are completed - events that take on the order of 10's of milliseconds - the protoneutron star cools by emitting a largely invisible radiation, a huge fluence 
of neutrinos of all flavors. Essentially all of the $3 \cdot 10^{53}$ ergs released in the collapse is radiated via electron, muon, and tauon neutrinos over the next 10 seconds.

In 1987 there existed deep underground two detectors consisting of tanks of ultrapure water instrumented with phototubes. They were originally constructed for the purpose of detecting proton decay, e.g. $p \rightarrow e^{+}+\gamma$, a process predicted by certain grand unified theories of elementary particle structure, but not yet observed. The $\gamma$ ray and Cerenkov light generated by the passage of the positron through the water would be detected by the phototubes, signaling a proton decay event. One detector was located in a Morton salt mine outside Cleveland, Ohio, while the second was on the other side of the globe in the Kamioka mine within the Japanese alps. While the search for a proton decay signal proved futile, on February 23, 1987 both detectors recorded a handful of events associated with the passage of a strong burst of antineutrinos through the tanks, a few of which initiated the reaction $\bar{\nu}_{e}+p \rightarrow e^{+}+n$. Within several hours a Canadian astronomer observing in Chile noted a new bright star. This was SN1987A.

How do these supernova neutrino events limit the neutrino mass? If a neutrino has mass, neutrinos of different energy will travel with different velocities according to

$$
v=\frac{p_{\nu}}{\sqrt{p_{\nu}^{2}+m_{\nu}^{2}}} \simeq 1-\frac{m_{\nu}^{2}}{2 E_{\nu}^{2}}
$$

rather than with the uniform velocity $c$. Consequently the time of arrival of higher energy neutrinos will be earlier than their lower energy counterparts. The difference in arrival times is then

$$
\frac{\delta t}{t} \sim \frac{\delta v}{v} \sim \frac{m_{\nu}^{2}}{E_{\nu}^{2}} \frac{\delta E_{\nu}}{E_{\nu}}
$$

where $t$ is the total time in transit from the Large Magellanic Cloud. The eleven events seen by the Kamiokande detector and the eight seen in Ohio have an energy spread $\delta E_{\nu} \sim 10 \mathrm{MeV}$ and an average energy $E_{\nu} 2-3$ times larger. The events arrived over a ten second time span. Thus we find in a simple analysis

$$
m_{\bar{\nu}_{e}} \lesssim E_{\nu}\left(\frac{\delta t}{t} \frac{E_{\nu}}{\delta E_{\nu}}\right)^{\frac{1}{2}} \sim 10 \mathrm{MeV}\left(\frac{10 \mathrm{sec}}{10^{13} \mathrm{sec}}\right)^{\frac{1}{2}} \sim 10 \mathrm{eV}
$$


A more careful analysis, which takes into account the time and energy distribution, yields a similar limit $-m_{\bar{\nu}_{e}} \lesssim 20 \mathrm{eV}$. It is remarkable that a handful of neutrino events from a star that lived and died long before the advent of civilization places a bound on the neutrino mass comparable to that gained from many years of high precision weak interactions studies!

We have focused on electron neutrino mass measurements because these have achieved the greatest precision. Bounds have also been established on the masses of the $\nu_{\mu}$ and $\nu_{\tau}$

$$
m_{\nu_{\mu}} \lesssim 170 \mathrm{keV}, \quad m_{\nu_{\tau}} \lesssim 24 \mathrm{MeV}
$$

from careful kinematic analyses of decays such as $\pi^{+} \rightarrow \mu^{+}+\nu_{\mu}$ and $\tau \rightarrow$ $5 \pi+\nu_{\tau}$. The short lifetimes of the $\pi$ and $\tau$ serve to limit the precision of the bounds. It is worth noting that some theories of neutrino mass predict that the $\nu_{\tau}$ will be the heaviest neutrino; e.g., in some models neutrino masses scale as the squares of the masses of the corresponding charged leptons. From this perspective the limits directly above are not necessarily less significant than the tighter bounds established on the $\bar{\nu}_{e}$.

This discussion can be succinctly summarized: there exists no evidence for massive neutrinos from direct measurements. Naturally, when increasingly precise experiments continue to yield values consistent with zero, it is tempting to assume that the number really is zero. Indeed, as mentioned above, in the so-called "standard model" of elementary particles, the neutrino mass is assumed to vanish. This does not follow from any fundamental principle, however, and could be modified accordingly if the evidence were to change, as we have noted before. That is precisely what has been happening over the last several years, as we will now describe.

\section{Probing Masses through Neutrino Mixing}

Significant improvements in the "direct" neutrino mass measurements described above will probably require considerable time and effort. For this reason there is great interest in measurements of a different type - those exploiting neutrino mixing - which might be able to probe far smaller masses, albeit in a less direct fashion. The essential idea can be traced to the seminal paper of Pontecorvo [26], who first pointed out that neutrino oscillations would occur if the neutrino states of definite mass do not coincide with the 
weak interaction eigenstates. To understand neutrino oscillations it is helpful to first consider the more familiar phenomenon of Faraday rotation, the rotation around the beam direction of the polarization vector of linearly polarized light as the light propagates through a magnetized material. Faraday rotation occurs because the index of refraction (and thereby the potential acting on the light) depends on the state of circular polarization-i.e., the two states of definite circular polarization $\left(\mid \chi_{ \pm}>=\sqrt{\frac{1}{2}}(|+x> \pm i|+y>)\right.$ evolve in time with distinct phases. Thus if $|\chi(t=0)>=|+x>=\sqrt{\frac{1}{2}}\left(\left|\chi_{+}>+\right| \chi_{-}>\right)$, then

$$
\begin{aligned}
\mid \chi(t)> & =\sqrt{\frac{1}{2}}\left(\left|\chi_{+}>\exp \left(-i \frac{\omega_{0}}{n_{+}} t\right)+\right| \chi_{-}>\exp \left(-i \frac{\omega_{0}}{n_{-}} t\right)\right) \\
& =\exp \left(-i \frac{\omega_{0}}{2} \frac{n_{+}+n_{-}}{n_{+} n_{-}} t\right)\left(\mid+x>\cos \left(\frac{\omega_{0}}{2} \frac{n_{+}-n_{-}}{n_{+} n_{-}} t\right)\right. \\
& \left.-\mid+y>\sin \left(\frac{\omega_{0}}{2} \frac{n_{+}-n_{-}}{n_{+} n_{-}} t\right)\right)
\end{aligned}
$$

so that the polarization vector rotates with frequency

$$
\omega_{p o l}=\frac{\omega_{0}}{2} \frac{n_{+}-n_{-}}{n_{+} n_{-}}
$$

Although we have three neutrino families, the essential physics of oscillations is illustrated very well by considering the interactions of only two of these, which we choose to be the $\nu_{e}$ and $\nu_{\mu}$. In this limit the situation is similar to the two-state Faraday rotation problem discussed above. The relevant part of the weak current involves the combination $\bar{e} \nu_{e}+\bar{\mu} \nu_{\mu}$ and is given by Eq. 13. This effectively defines the $\nu_{e}$ and $\nu_{\mu}$ : they are the neutrino weak interaction eigenstates, the neutrinos accompanying the electron and muon, respectively, when these particles are weakly produced. Yet there is a second Hamiltonian, the free Hamiltonian describing the propagation of an isolated neutrino. The eigenstates of this Hamiltonian are the mass eigenstates. If the two mass eigenstates are distinct (and thus at least one is nonzero), then in general the eigenstates diagonalizing the mass Hamiltonian will not diagonalize the weak interaction. If we label the mass eigenstates as $\mid \nu_{1}>$ and $\mid \nu_{2}>$, then

$$
\begin{aligned}
& \left|\nu_{1}>=\cos \theta\right| \nu_{e}>+\sin \theta \mid \nu_{\mu}>\quad \text { with mass } m_{1} \\
& \left|\nu_{2}>=-\sin \theta\right| \nu_{e}>+\cos \theta \mid \nu_{\mu}>\quad \text { with mass } m_{2}
\end{aligned}
$$


where $\theta$ is a mixing angle which distinguishes the mass and weak eigenstates. Also suppose that at time $t=0$ an electron neutrino is produced with fixed momentum $\vec{p}$

$$
|\psi(t=0)>=| \nu_{e}>=\cos \theta\left|\nu_{1}>-\sin \theta\right| \nu_{2}>
$$

The mass eigenstates propagate with simple phases, as they are the eigenstates of the free Hamiltonian. At a distance $\sim c t$ from the source the neutrino state is

$$
|\psi(t)>=\cos \theta| \nu_{1}>e^{-i E_{1} t}-\sin \theta \mid \nu_{2}>e^{-i E_{2} t}
$$

where $E_{i}=\sqrt{p^{2}+m_{i}^{2}}$. Projecting back upon weak eigenstates, we have

$$
\begin{aligned}
& <\nu_{e} \mid \psi(t)>=\cos ^{2} \theta e^{-i E_{1} t}+\sin ^{2} \theta e^{-i E_{2} t} \\
& <\nu_{\mu} \mid \psi(t)>=\cos \theta \sin \theta\left(e^{-i E_{1} t}-e^{-i E_{2} t}\right)
\end{aligned}
$$

Then, noting that

$$
\begin{aligned}
& E_{1} \sim p+\frac{m_{1}^{2}+m_{2}^{2}}{4 p}-\frac{\delta m^{2}}{4 p} \\
& E_{2} \sim p+\frac{m_{1}^{2}+m_{2}^{2}}{4 p}+\frac{\delta m^{2}}{4 p}
\end{aligned}
$$

where $\delta m^{2}=m_{2}^{2}-m_{1}^{2}$, we find at $t>0$ a probability

$$
p(t)=\left|<\nu_{\mu}\right| \psi(t)>\left.\right|^{2}=\sin ^{2} 2 \theta \sin ^{2} \frac{\delta m^{2} t}{4 p}
$$

that the $\nu_{e}$ will have transformed into a $\nu_{\mu}$. This change of neutrino identity is called a "neutrino oscillation," due to the time- or distance-dependent oscillation in $p(t)$. This phenomenon is a sensitive test of neutrino masses given nondegenerate neutrinos and a nonzero mixing angle $\theta$. As the standard electroweak model describes neutrinos as massless, the observation of neutrino oscillations would constitute definitive evidence of physics beyond the standard model.

Three sources of neutrinos have been exploited by experimentalists seeking (and apparently finding!) neutrino oscillations: 


\subsection{Accelerator and Reactor Experiments}

Such experiments exploit an accelerator or reactor to produce a large flux of neutrinos/antineutrinos. Measurements are then done downstream to determine whether the character of the neutrinos has changed. Such experiments fall into one of two categories. In the first-called "disappearance" experiments - one looks for deviations in the expected flux of neutrinos of a definite type. For example, the flux from a reactor is overwhelmingly of the $\bar{\nu}_{e}$ type. Thus oscillations into a second flavor would lead to an unexpected reduction in the flux some distance downstream, which might be monitored through reactions such as $\bar{\nu}_{e}+p \rightarrow e^{+}+n$. Even if the reactor flux were poorly understood, a definitive signal could result from detectors placed at different distances $L_{1}$ and $L_{2}$ from the reactor, as shown in Fig. 6a. In the absence of oscillations the flux must fall off as $1 / L^{2}$ (apart from corrections due to the finite size of the source). However oscillations superimpose an additional factor of $1-p(t)$, leading to a modulation that clearly signals the "new physics." The second type of oscillation experiment, called an "appearance" experiment, is a search for the product of the oscillation. For example, at an accelerator like the Los Alamos Meson Physics Facility (LAMPF), proton interactions in the beam stop produce large numbers of $\nu_{\mu}$ 's, $\bar{\nu}_{\mu}$ 's, and $\nu_{e}$ 's, but very few $\bar{\nu}_{e}$ 's: the reaction chains involve $\pi^{+} \rightarrow \mu^{+}+\nu_{\mu}$ followed by $\mu^{+} \rightarrow e^{+}+\nu_{e}+\bar{\nu}_{\mu}$, or $\pi^{-} \rightarrow \mu^{-}+\bar{\nu}_{\mu}$ followed by $\mu^{-}+(A, Z) \rightarrow(A, Z-1)+\nu_{\mu}$. It is the importance of this last reaction that prevents the $\mu^{-}$from ondergoing the free decay $\mu^{-} \rightarrow e^{-}+\bar{\nu}_{e}+\nu_{\mu}$-negative muons are slowed by their interactions with nuclei in the beam stop and captured into atomic orbitals, then rapidly cascade electromagnetically into the $1 s$ orbital. There the $\mu^{-}$ wave function has a strong overlap with the nucleus (especially for the high $\mathrm{Z}$ nuclei in the beam stop), allowing the $\mu^{-}$capture to proceed much more rapidly than the free decay. Thus there are very few electron-type antineutrinos 27] and a sensitive search for the flavor oscillation $\bar{\nu}_{\mu} \rightarrow \bar{\nu}_{e}$ can be done by looking for the appearance of a flux of $\bar{\nu}_{e}$ 's downstream from the beam

stop, as shown in Figure 6b. Such neutrinos can be efficiently detected via the reaction $\bar{\nu}_{e}+p \rightarrow n+e^{+}$. The rate should depend on $1 / L^{2}$ modulated by $p(t)$.

The results of such experiments are generally presented in terms of a two-dimensional diagram with $\delta m^{2}$ plotted against $\sin ^{2} 2 \theta$. Of course, if no signal is observed in such an experiment it does not necessarily imply that 


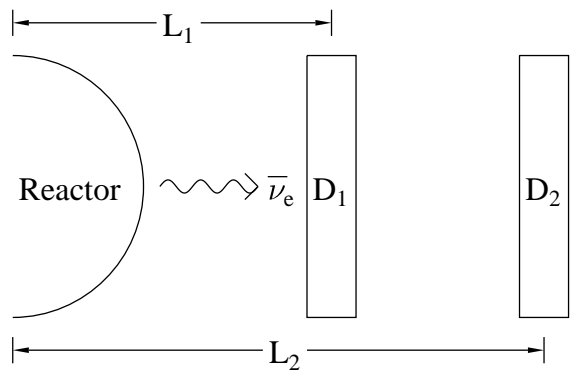

a)

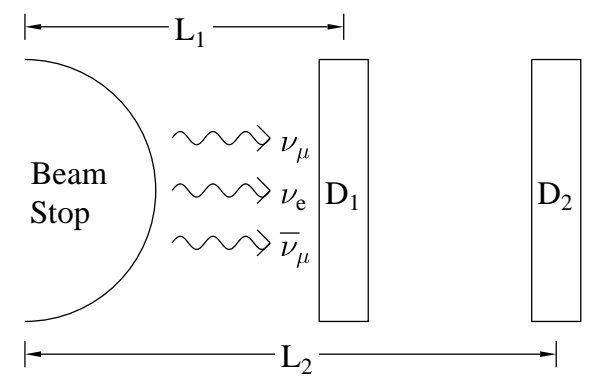

b)

Figure 6: a) A neutrino oscillation experiment of the "disappearance" typea flux of reactor antineutrinos is monitored by detectors at various distances from the source - in which deviations from a simple $1 / L^{2}$ falloff are sought; b) A neutrino oscillation of the "appearance" type: detectors are placed at various distances from a beam stop neutrino source in order to detect the appearance of an oscillatory $\bar{\nu}_{e}$ component.

no oscillations are occurring. It could be that the mixing angle $\theta$ is very tiny (and thus $p(t)$ too small to be detected), or that $\delta m^{2}$ is too small (so that effects appear only at distances $L$ so large that the neutrino flux has fallen below detectable limits). In order to quantify this assertion note that we can write the oscillation probabilty in terms of path length

$$
p(t) \simeq \sin ^{2} 2 \theta \sin ^{2} \frac{\delta m^{2} L}{4 E_{\nu}}
$$

which shows that the maximum effect occurs for

$$
\frac{\delta m^{2} L}{4 E_{\nu}}=\frac{\pi}{2} \Rightarrow L=\frac{2 \pi E_{\nu}}{\delta m^{2}}
$$

Thus a smaller $\delta m^{2}$ implies a longer oscillation length, so that a more intense neutrino source is required to combate the associated $1 / L^{2}$ fall off in the flux. Experiments have ruled out a large portion of the $\sin ^{2} 2 \theta-\delta m^{2}$ space, e.g., $\sin ^{2} 2 \theta \gtrsim 0.01$ and $\delta m^{2} \gtrsim 0.1 \mathrm{eV}^{2}$, as shown in Figure 7 . However one experiment-LSND at Los Alamos - has claimed a positive signal 
for neutrino oscillations. This experiment, situated downstream from the LAMPF beamstop, uses a 52,000 gallon tank of mineral oil and a small amount of liquid scintillator instrumented with 1220 phototubes. A neutrino event $\bar{\nu}_{e}+p \rightarrow n+e^{+}$is indicated by a combination of Cerenkov and scintillator light produced by the positron followed (after a couple of hundred microseconds) by a $2.2 \mathrm{MeV}$ gamma ray from the capture of the produced neutron, $n+p \rightarrow d+\gamma$. Only 0.0000000001 percent of the LAMPF neutrinos interact in the tank. Thus the challenge is to distinguish this tiny signal from the $3 \times 10^{8}$ cosmic rays which pass through the tank each day. After very careful numerical simulations, the LSND collaboration announced they had detected $22 \bar{\nu}_{e}$ events compared to an anticipated background of $4.6 \pm 0.6$ events [28]. This excess of events is consistent with neutrino oscillations for the values of $\delta m^{2}$ and $\sin ^{2} 2 \theta$ shown in Figure 7: the narrow allowed region includes the ranges $\delta m^{2} \sim 0.2-2 \mathrm{eV}^{2}$ and $\sin ^{2} 2 \theta \sim 0.03-0.003$. Since publication of the first paper, the collaboration's data set has grown to $\sim$ 60 events. Concurrently, the competing KARMEN group of the Rutherford Laboratory in England has found no such oscillation signal, though the sensitivity achieved to date leaves a substantial portion of the LSND allowed $\delta m^{2}-\sin ^{2} 2 \theta$ region untested. An improved experiment at Fermilab, which should have thousands of events if LSND is correct, has recently been approved and should yield results by 2002 .

\subsection{Atmospheric Neutrinos}

When high energy cosmic rays strike the earth's atmosphere a multitude of secondary particles are produced, most of which travel at nearly the speed of light in the same direction as the incident cosmic ray. Many of the secondaries are pions and kaons, which decay as described above into electrons, muons, and electron and muon neutrinos and antineutrinos. These neutrinos reach and pass through the earth. The fluxes are large: about a hundred such cosmic ray-induced neutrinos pass through each of us every second. Yet because these particles react weakly, only one interaction is expected per human body every thousand years! Thus a considerably larger target is required for a reasonable event rate. In SuperKamiokande, the massive 50,000 ton water detector that replaced the original 3000 ton Kamiokande detector, one event occurs every 90 minutes. The energies of these neutrinos (typically $1 \mathrm{GeV}$ ) are sufficiently high to produce either electrons or muons, 


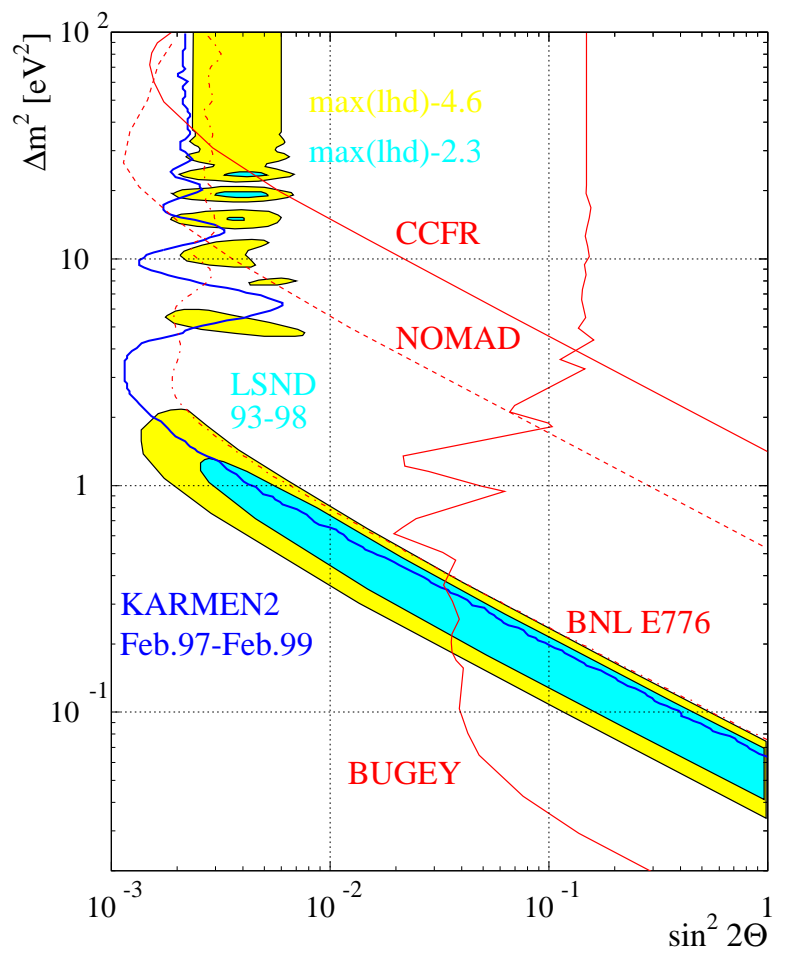

Figure 7: Regions of $\delta m^{2}$ and $\sin ^{2} 2 \theta$ ruled out by various null experiments are shown along with the allowed region corresponding to the LSND results. Thus the portion of the LSND region below the KARMEN exclusion region and to the left of the Bugey exclusion region gives the candidate oscillation parameters. This figure was provided by Bill Louis. 
depending on the neutrino flavor. As these charged particles pass through the water, they produce Cerenkov radiation. However, the Cerenkov ring produced by an energetic electron is more diffuse than the relatively clean ring of a muon. This allows the experimenters to distinguish electrons from muons with about $98 \%$ accuracy. Since the charged lepton tends to travel in the same direction as the incident neutrino, the experimenters can thus deduce both the flavor and the direction of neutrinos that react in the water.

A decade ago it was already apparent that atmospheric neutrino rates seen in existing detectors were anomalous. Using known cross sections and decay rates theorists had predicted about twice as many muon neutrinos as electron neutrinos from cosmic ray events. For example, we mentioned previously that a $\pi^{+}$decays into an $e^{+}$, a $\nu_{e}$, a $\nu_{\mu}$, and a $\bar{\nu}_{\mu}$. That is, two muon neutrinos are produced, but only one $\nu_{e}$. However, most of the early atmospheric neutrino experiments found the electron-to-muon ratio from neutrino reactions to be approximately unity. The very precise measurements made with SuperKamiokande appear to show that the ratio has this unexpected value because of a deficit in muon-like events - the electron event rate is about as expected. The muon deficit has a strong zenith angle dependence, with the largest suppression associated with atmospheric neutrinos coming from below, e.g., originating on the opposite side of the earth. Such a dependence of the muon-to-electron ratio on distance is a signature of neutrino oscillations, as we have noted. The most plausible interpretation of the SuperKamiokande data 29] is that atmospheric $\nu_{\mu}$ 's are oscillating into $\nu_{\tau}$ 's, which are not observed because the $\nu_{\tau}$ 's are too low in energy to produce $\tau$ 's in SuperKamiokande. The strong suppression in the $\nu_{\mu}$ flux is characteristic of maximal mixing $(\theta \sim \pi / 4)$, while the zenith angle dependence indicates that the oscillation length is comparable to the earth's diameter. The corresponding $\delta m^{2}$ is $\sim 2 \cdot 10^{-3} \mathrm{eV}^{2}$. As many theoretical models predict the $\nu_{\tau}$ to be significantly heavier than the $\nu_{\mu}$, this suggests a $\nu_{\tau}$ mass of $\sim 0.05 \mathrm{eV}$. The quality of the SuperKamiokande data - the statistical error on the muon-to-electron event rate is well below $10 \%$ and there is remarkable consistency between the sub-GeV and multi-GeV data sets and between the fully- and partially-contained data sets - provides a powerful argument that oscillations have been observed. Because the zenith-angle dependence shows that the $\nu_{\mu}$ flux depends on distance, the atmospheric data provide direct proof of oscillations. Thus this may be our strongest evidence for massive neutrinos and for the incompleteness of the standard model. 
There is another remarkable aspect of the atmospheric neutrino results. If neutrinos are massive, there must be some reason that their masses are so much lighter than those of all the more familiar quarks and leptons. In fact, a lovely explanation is provided in many proposed extensions of the standard model that again returns to the idea that neutrinos are special because they can have both Dirac and Majorana masses. The explanation is called the seesaw mechanism [30]. It predicts that the neutrino mass is

$$
m_{\nu}=m_{D}\left(\frac{m_{D}}{m_{R}}\right)
$$

where $m_{D}$ is a Dirac mass often equated to the mass of the corresponding quark or charged lepton, while $m_{R}$ is a very heavy Majorana mass associated with interactions at energies far above the reach of existing accelerators. This seesaw mechanism arises naturally in models with both Dirac and Majorana masses. In the case of the $\nu_{\tau}$ we concluded from the atmospheric neutrino data that its mass might be $\sim 0.05 \mathrm{eV}$. A reasonable choice for $m_{D}$ is the mass of the corresponding third generation quark, the top quark, $m_{D} \sim 200$ $\mathrm{GeV}$. It follows that $m_{R} \sim 10^{14} \mathrm{GeV}$ ! Thus tiny neutrino masses might be our window on physics at enormous energy scales. This large mass $m_{R}$ is interesting because there is an independent argument, based on observations that the weak, electromagnetic, and strong interactions would all have approximately the same strength at $\sim 10^{16} \mathrm{GeV}$, that suggests a very similar value for the "grand unification scale." This has led many in the community to hope that the pattern of neutrino masses now being discovered may help us probe the structure of the theory that lies beyond the standard model [31].

\subsection{Solar Neutrinos}

The thermonuclear reactions occurring in its core make the sun a marvelous source of neutrinos of a single flavor, $\nu_{e}$. The standard solar model - really, the standard model of main sequence stellar evolution - allows us to predict the flux and spectrum of these neutrinos. The standard solar model makes four basic assumptions:

- The sun evolves in hydrostatic equilibrium, maintaining a local balance between the gravitational force and the pressure gradient. To describe this 
condition, one must specify the equation of state as a function of temperature, density, and composition.

- Energy is transported by radiation and convection. While the solar envelope is convective, radiative transport dominates in the core region where thermonuclear reactions take place. The opacity depends sensitively on the solar composition, particularly the abundances of heavier elements.

- Solar energy is produced by thermonuclear reaction chains in which four protons are converted to ${ }^{4} \mathrm{He}$

$$
4 \mathrm{p} \rightarrow{ }^{4} \mathrm{He}+2 \mathrm{e}^{+}+2 \nu_{\mathrm{e}}
$$

The standard solar model predicts that $98 \%$ of these reactions occur through the pp chain illustrated in Figure 8, with the CNO cycle accounting for the remainder. The sun is a slow reactor, characterized by a relatively low core temperature $T_{c} \sim 1.5 \cdot 10^{7} \mathrm{~K}$. Thus Coulomb barriers tend to suppress the rates of reactions involving higher $\mathrm{Z}$ nuclei, an effect we will see reflected in the neutrino fluxes given below.

- The model is constrained to produce today's solar radius, mass, and luminosity. An important assumption of the standard solar model is that the sun was highly convective, and therefore uniform in composition, when it first entered the main sequence. It is furthermore assumed that the surface abundances of metals (nuclei heavier than $\mathrm{He}$ ) were undisturbed by the sun's subsequent evolution, and thus provide a record of the initial core metallicity. The remaining parameter is the initial ${ }^{4} \mathrm{He} / \mathrm{H}$ ratio, which is adjusted until the model reproduces the known solar luminosity at the sun's present age, 4.6 billion years.

Figure 8 shows that the pp chain is comprised of three distinct cycles, each of which is tagged by a distinctive neutrino. The total rate of ppI+ppII+ppIII burning is governed by the rate at which protons are consumed

$$
\mathrm{p}+\mathrm{p} \rightarrow{ }^{2} \mathrm{H}+\mathrm{e}^{+}+\nu_{\mathrm{e}}
$$

a reaction which produces an allowed $\beta$ decay spectrum (that is, a spectrum like that of Eq. 5) of low-energy (0.42 MeV endpoint) $\nu_{e}$ 's. The ppII rate is tagged by the distinctive neutrino lines from electron capture on ${ }^{7} \mathrm{Be}(0.86$ and $0.36 \mathrm{MeV})$. Finally the ppIII cycle is tagged by the high-energy neutrinos from the $\beta$ decay of ${ }^{8} \mathrm{~B}(\sim 15 \mathrm{MeV}$ endpoint). The competition between 


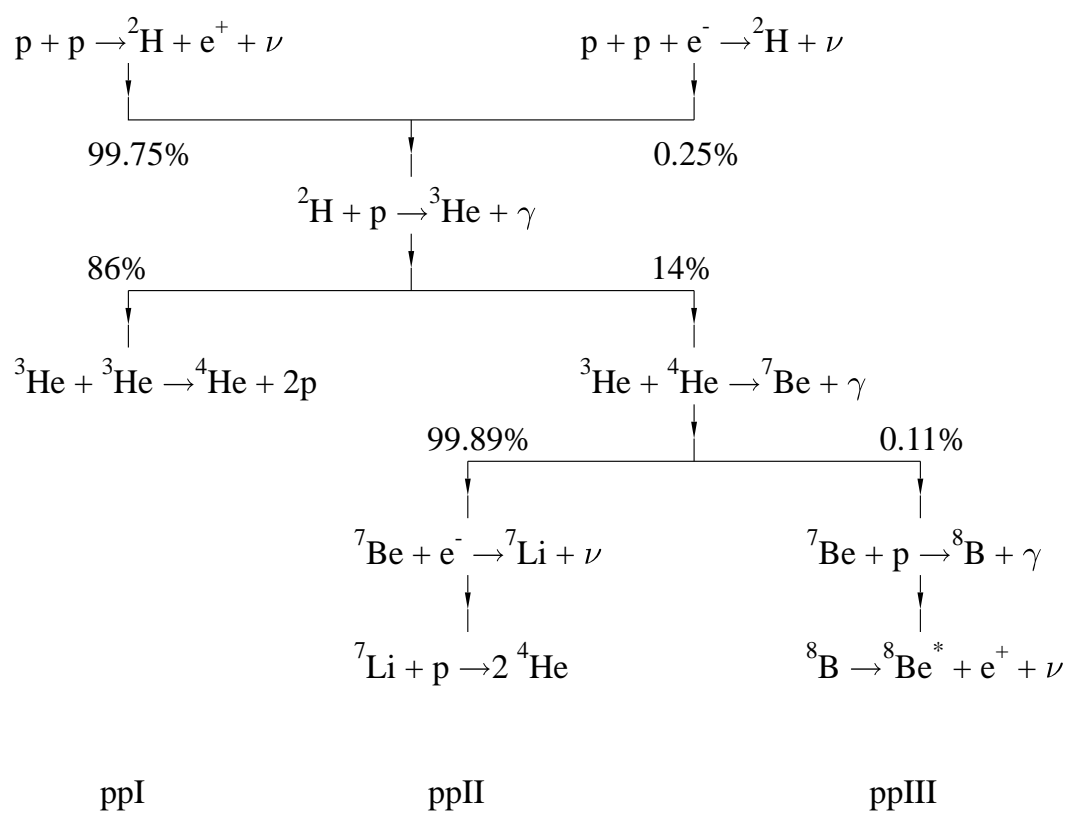

Figure 8: The three cycles comprising the pp chain.

these three cycles depends sensitively on the solar core temperature $T_{c}$. Thus the original motivation for measuring solar neutrinos was to determine the relative rates of the ppI, ppII, and ppIII cycles, from which the core temperature could be deduced to an accuracy of a few percent, thereby checking the standard solar model.

The neutrino flux predictions of the standard solar model are summarized below [32].

$$
\begin{array}{lll}
\text { Reaction } & E_{\nu}^{\max } & \operatorname{Flux}\left(10^{10} / \mathrm{cm}^{2} / \mathrm{sec}\right) \\
\mathrm{p}+\mathrm{p} \rightarrow{ }^{2} \mathrm{H}+e^{+}+\nu_{e} & 0.42 & 5.94 \\
{ }^{7} \mathrm{Be}+e^{-} \rightarrow{ }^{7} \mathrm{Li}+\nu_{e} & 0.86(90 \%) & 4.80 \times 10^{-1} \\
& 0.36(10 \%) & \\
{ }^{8} \mathrm{~B} \rightarrow{ }^{8} \mathrm{Be}^{*}+e^{+}+\nu_{e} & 14.06 & 5.15 \times 10^{-4}
\end{array}
$$

The first experiment to test these predictions began more than three decades ago with a detector placed a mile underground in the Homestake Gold Mine in Lead, South Dakota. This great depth protects the detector from all forms of cosmic radiation other than neutrinos. Ray Davis, Jr., and 
his collaborators [33] filled the detector with 615 tons of the cleaning fluid perchloroethylene $\left(\mathrm{C}_{2} \mathrm{Cl}_{4}\right)$ in order to make use of the reaction

$$
{ }^{37} \mathrm{Cl}\left(\nu_{\mathrm{e}}, \mathrm{e}^{-}\right){ }^{37} \mathrm{Ar}
$$

The few atoms of the noble gas ${ }^{37} \mathrm{Ar}$ produced in the tank after a typical $(\sim$ two month) exposure could be recovered quantitatively by a helium purge, then counted via the subsequent electron capture reaction $e^{-}+{ }^{37} \mathrm{Ar} \rightarrow{ }^{37} \mathrm{Cl}$ $+\nu_{e}$, which has a 35-day half life. The miniscule ${ }^{37} \mathrm{Ar}$ production rate, less than an atom every two days, has been measured to an accuracy of better than $10 \%$ by patient effort. The deduced solar neutrino capture rate, 2.56 $\pm 0.16 \pm 0.16 \mathrm{SNU}\left(1 \mathrm{SNU}=10^{-36}\right.$ captures/atom/sec) is about $1 / 3$ of the standard solar model prediction. As this reaction is primarily sensitive to ${ }^{8} \mathrm{~B}(78 \%)$ and ${ }^{7} \mathrm{Be}(15 \%)$ neutrinos, one concludes that the sun is producing fewer high energy neutrinos than expected.

Similar radiochemical experiments were done by the SAGE and GALLEX collaborations [35] using a different target, one containing ${ }^{71} \mathrm{Ga}$. The special properties of this nucleus include an unusually low threshold for the reaction ${ }^{71} \mathrm{Ga}\left(\nu_{e}, e^{-}\right){ }^{71} \mathrm{Ge}$, leading to a large cross section for the capture of low-energy pp neutrinos. The resulting experimental capture rates are $66 \pm 13 \pm 6$ and $76 \pm 8$ SNU for the SAGE and GALLEX detectors, respectively, which can be compared to the standard solar model prediction of $\sim 130$ SNU. Most important, as the pp flux is directly constrained by the rate of hydrogen burning and thus by the observed solar luminosity in all steady-state solar models, there is a minimum theoretical value for the capture rate of 79 SNU, given standard model weak interaction physics. With this assumption, it appears that there is virtually no contribution from ppII and ppIII cycle neutrinos.

The remaining experiments, Kamiokande II/III and the ongoing SuperKamiokande [34], exploit water Cerenkov detectors to view solar neutrinos on an event-by-event basis. Just as described in our atmospheric neutrino discussion, the scattering of high energy ${ }^{8} \mathrm{~B}$ neutrinos produces recoil electrons and thus Cerenkov radiation that can be recorded in the surrounding phototubes. The correlation of the electron direction with the position of the sun is crucial in separating solar neutrino events from background. After 504 days of operations the rate measured by SuperKamiokande, which under current operating conditions is sensitive to neutrinos with energies above $\sim 6$ 
$\mathrm{MeV}$, is consistent with an ${ }^{8} \mathrm{~B}$ neutrino flux of $\left(2.44 \pm 0.05_{-0.07}^{+0.09}\right) \cdot 10^{6} / \mathrm{cm}^{2} \mathrm{sec}$. This is about half of the standard solar model prediction.

If one combines the various experimental results and assumes that the neutrino spectra are not being distorted by oscillations or other new physics, the following pattern of fluxes emerges

$$
\begin{aligned}
\phi(p p) & \sim 0.9 \phi^{\mathrm{SSM}}(p p) \\
\phi\left({ }^{7} \mathrm{Be}\right) & \sim 0 \\
\phi\left({ }^{8} \mathrm{~B}\right) & \sim 0.4 \phi^{\mathrm{SSM}}\left({ }^{8} \mathrm{~B}\right)
\end{aligned}
$$

where SSM stands for the standard solar model. In fact, the preferred value of $\phi\left({ }^{7} \mathrm{Be}\right.$ ) turns out to be negative (at 2-3 $\sigma$ ) in unconstrained fits. A reduced ${ }^{8} \mathrm{~B}$ neutrino flux can be produced by lowering the central temperature of the sun somewhat, as $\phi\left({ }^{8} \mathrm{~B}\right) \sim T_{c}^{18}$. However, such an adjustment, either by varying the parameters of the standard solar model or by adopting some nonstandard physics, tends to push the $\phi\left({ }^{7} \mathrm{Be}\right) / \phi\left({ }^{8} \mathrm{~B}\right)$ ratio to higher values rather than the low one required by the results above,

$$
\frac{\phi\left({ }^{7} \mathrm{Be}\right)}{\phi\left({ }^{8} \mathrm{~B}\right)} \sim T_{c}^{-10}
$$

Thus the observations seem difficult to reconcile with plausible solar model variations: one observable, $\phi\left({ }^{8} \mathrm{~B}\right)$, requires a cooler core while a second, the ratio $\phi\left({ }^{7} \mathrm{Be}\right) / \phi\left({ }^{8} \mathrm{~B}\right)$, requires a hotter one.

These arguments seem to favor a more radical solution, one involving new properties of neutrinos. Originally the most plausible such solution was neutrino oscillations of the type we discussed in connection with atmospheric neutrinos and LSND. For simplicity we consider the mixing of the $\nu_{e}$ with a single second flavor, the $\nu_{\mu}$. This will clearly alter the expectation for solar neutrino experiments, as the Homestake and SAGE/GALLEX experiments cannot detect $\nu_{\mu}$ 's, while SuperKamiokande detects $\nu_{\mu}$ 's with reduced efficiency. (The cross section for $\nu_{\mu}$ scattering off electrons is about $1 / 6$ that for $\nu_{e}$ scattering.) The $\nu_{e}$ survival probability at the earth is

$$
p_{\nu_{e}}(x)=1-\sin ^{2}(2 \theta) \sin ^{2}\left(\frac{\delta m^{2} c^{4} x}{4 \hbar c E}\right) \sim 1-\frac{1}{2} \sin ^{2}(2 \theta)
$$

where $\theta$ is the $\nu_{e}-\nu_{\mu}$ mixing angle. (This is just our earlier result of Eq. 65, with the replacement $p \sim E$ and with the factors of $c$ and $\hbar$ reinserted.) The 
result on the right is appropriate if the oscillation length $L_{0}=4 \pi \hbar c E / \delta m^{2} c^{4}$ is much smaller that the earth-sun distance $x$. In that case (for a broad spectrum such as the ${ }^{8} \mathrm{~B}$ neutrinos) the oscillatory factor averages to $1 / 2$. For ${ }^{8} \mathrm{~B}$ neutrinos this averaging is appropriate if $\delta m^{2} c^{4}$ exceeds $10^{-9} \mathrm{eV}^{2}$.

Even if the mixing angle were maximal $-\theta \sim \pi / 4-$ such vacuum oscillations would then produce a factor of two suppression in the neutrino flux, not the factor of three indicated by the ${ }^{37} \mathrm{Cl}$ result. Historically large mixing angles were viewed as contrived, as the similar Cabibbo angle describing the weak mixing of quarks is quite small. The recent atmospheric neutrino results, which strongly favor large mixing angles, have weakened this argument. Nevertheless, one of the marvelous properties of our sun is that it can greatly enhance oscillations even if mixing angles are quite small. We now turn to describing this effect, which is known as the Mihkeyev-Smirnov-Wolfenstein mechanism.

The starting point is a slight generalization of our vacuum neutrino oscillation discussion. Previously we discussed the case where our initial neutrino had a definite flavor. But we could have considered the somewhat more general case

$$
|\nu(t=0)\rangle=a_{e}(t=0)\left|\nu_{e}\right\rangle+a_{\mu}(t=0)\left|\nu_{\mu}\right\rangle .
$$

Exactly as before we could expand this wave function in terms of the mass eigenstates, which propagate simply, to find (this takes a bit of algebra)

$$
i \frac{d}{d x}\left(\begin{array}{c}
a_{e} \\
a_{\mu}
\end{array}\right)=\frac{1}{4 E}\left(\begin{array}{cc}
-\delta m^{2} \cos 2 \theta_{v} & \delta m^{2} \sin 2 \theta_{v} \\
\delta m^{2} \sin 2 \theta_{v} & \delta m^{2} \cos 2 \theta_{v}
\end{array}\right)\left(\begin{array}{l}
a_{e} \\
a_{\mu}
\end{array}\right) .
$$

Note that the common phase has been ignored: it can be absorbed into the overall phase of the coeficients $a_{e}$ and $a_{\mu}$, and thus has no consequence. We have also labeled the mixing angle as $\theta_{v}$, to emphasize that it is the vacuum value, and equated $x=t$, that is, set $c=1$.

The view of neutrino oscillations changed when Mikheyev and Smirnov [36] showed in 1985 that the density dependence of the neutrino effective mass, a phenomenon first discussed by Wolfenstein [37] in 1978, could greatly enhance oscillation probabilities: a $\nu_{e}$ is adiabatically transformed into a $\nu_{\mu}$ as it traverses a critical density within the sun. It became clear that the sun was not only an excellent neutrino source, but also a natural regenerator for cleverly enhancing the effects of flavor mixing. 
The effects of matter alter our neutrino evolution equation in an apparently simple way

$$
i \frac{d}{d x}\left(\begin{array}{c}
a_{e} \\
a_{\mu}
\end{array}\right)=\frac{1}{4 E}\left(\begin{array}{cc}
2 E \sqrt{2} G_{F} \rho(x)-\delta m^{2} \cos 2 \theta_{v} & \delta m^{2} \sin 2 \theta_{v} \\
\delta m^{2} \sin 2 \theta_{v} & -2 E \sqrt{2} G_{F} \rho(x)+\delta m^{2} \cos 2 \theta_{v}
\end{array}\right)\left(\begin{array}{c}
a_{e} \\
a_{\mu}
\end{array}\right)
$$

where $\rho(x)$ is the solar electron density. The new contribution to the diagonal elements, $2 E \sqrt{2} G_{F} \rho(x)$, represents the effective contribution to $m_{\nu}^{2}$ that arises from neutrino-electron scattering. The indices of refraction of electron and muon neutrinos differ because the former scatter by charged and neutral currents, while the latter have only neutral current interactions: the sun contains electrons but no muons. The difference in the forward scattering amplitudes determines the density-dependent splitting of the diagonal elements of the new matter equation.

It is helpful to rewrite this equation in a basis consisting of the light and heavy local mass eigenstates (i.e., the states that diagonalize the right-hand side of the equation),

$$
\begin{aligned}
\left|\nu_{L}(x)\right\rangle & =\cos \theta(x)\left|\nu_{e}\right\rangle-\sin \theta(x)\left|\nu_{\mu}\right\rangle \\
\left|\nu_{H}(x)\right\rangle & =\sin \theta(x)\left|\nu_{e}\right\rangle+\cos \theta(x)\left|\nu_{\mu}\right\rangle .
\end{aligned}
$$

The local mixing angle is defined by

$$
\begin{aligned}
\sin 2 \theta(x) & =\frac{\sin 2 \theta v}{\sqrt{X^{2}(x)+\sin ^{2} 2 \theta v}} \\
\cos 2 \theta(x) & =\frac{-X(x)}{\sqrt{X^{2}(x)+\sin ^{2} 2 \theta v}}
\end{aligned}
$$

where $X(x)=2 \sqrt{2} G_{F} \rho(x) E / \delta m^{2}-\cos 2 \theta_{v}$. Thus $\theta(x)$ ranges from $\theta_{v}$ to $\pi / 2$ as the density $\rho(x)$ goes from 0 to $\infty$.

If we define

$$
|\nu(x)\rangle=a_{H}(x)\left|\nu_{H}(x)\right\rangle+a_{L}(x)\left|\nu_{L}(x)\right\rangle
$$

the neutrino propagation can be rewritten in terms of the local mass eigenstates

$$
i \frac{d}{d x}\left(\begin{array}{c}
a_{H} \\
a_{L}
\end{array}\right)=\left(\begin{array}{cc}
\lambda(x) & i \alpha(x) \\
-i \alpha(x) & -\lambda(x)
\end{array}\right)\left(\begin{array}{c}
a_{H} \\
a_{L}
\end{array}\right)
$$


with the splitting of the local mass eigenstates determined by

$$
2 \lambda(x)=\frac{\delta m^{2}}{2 E} \sqrt{X^{2}(x)+\sin ^{2} 2 \theta v}
$$

and with mixing of these eigenstates governed by the density gradient

$$
\alpha(x)=\left(\frac{E}{\delta m^{2}}\right) \frac{\sqrt{2} G_{F} \frac{d}{d x} \rho(x) \sin 2 \theta_{v}}{X^{2}(x)+\sin ^{2} 2 \theta_{v}} .
$$

The results above are quite interesting: the local mass eigenstates diagonalize the matrix if the density is constant, that is, if $\alpha=0$. In such a limit, the problem is no more complicated than our original vacuum oscillation case, although our mixing angle is changed because of the matter effects. But if the density is not constant, the mass eigenstates in fact evolve as the density changes. This is the crux of the MSW effect. Note that the splitting achieves its minimum value, $\frac{\delta m^{2}}{2 E} \sin 2 \theta_{v}$, at a critical density $\rho_{c}=\rho\left(x_{c}\right)$

$$
2 \sqrt{2} E G_{F} \rho_{c}=\delta m^{2} \cos 2 \theta_{v}
$$

that defines the point where the diagonal elements of the matrix in Eq. 78 cross.

Our local-mass-eigenstate form of the propagation equation can be trivially integrated if the splitting of the diagonal elements is large compared to the off-diagonal elements, so that the effects of $\alpha(x)$ can be ignored

$$
\gamma(x)=\left|\frac{\lambda(x)}{\alpha(x)}\right|=\frac{\sin ^{2} 2 \theta_{v}}{\cos 2 \theta_{v}} \frac{\delta m^{2}}{2 E} \frac{1}{\left|\frac{1}{\rho_{c}} \frac{d \rho(x)}{d x}\right|} \frac{\left[X(x)^{2}+\sin ^{2} 2 \theta_{v}\right]^{3 / 2}}{\sin ^{3} 2 \theta_{v}} \gg 1,
$$

a condition that becomes particularly stringent near the crossing point, where $X(x)$ vanishes,

$$
\gamma_{c}=\gamma\left(x_{c}\right)=\frac{\sin ^{2} 2 \theta_{v}}{\cos 2 \theta_{v}} \frac{\delta m^{2}}{2 E} \frac{1}{\left|\frac{1}{\rho_{c}} \frac{d \rho(x)}{d x}\right|_{x=x_{c}} \mid} \gg 1 .
$$

The resulting adiabatic electron neutrino survival probability [38], valid when $\gamma_{c} \gg 1$, is

$$
p_{\nu_{e}}^{\text {adiab }}=\frac{1}{2}+\frac{1}{2} \cos 2 \theta_{v} \cos 2 \theta_{i}
$$




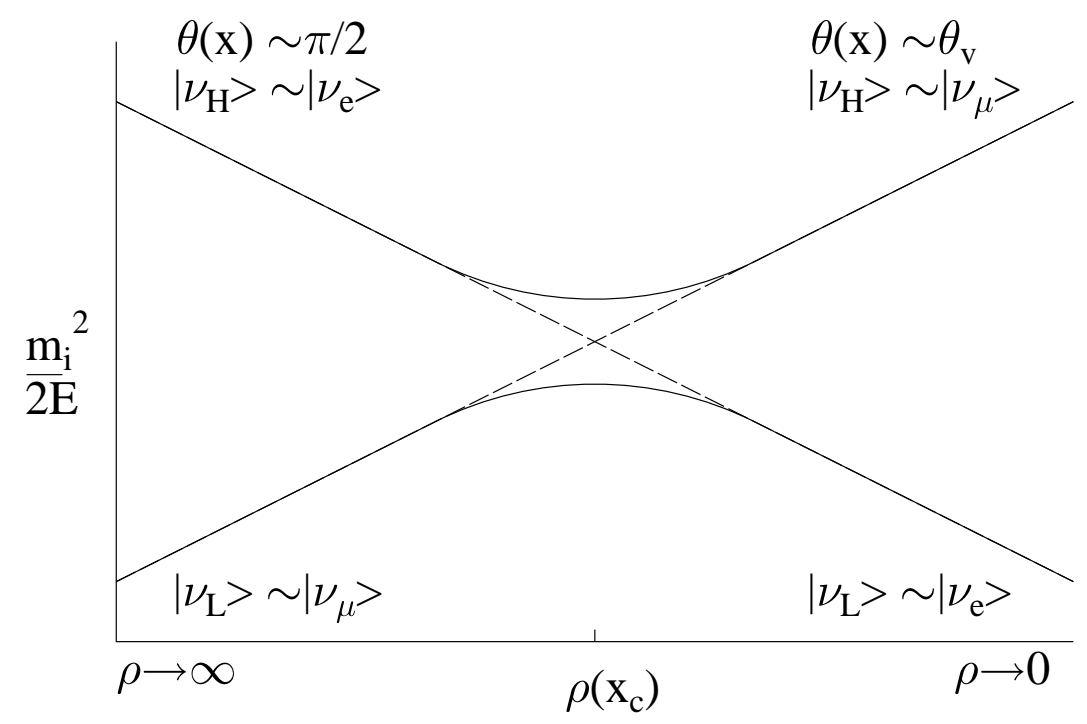

Figure 9: Schematic illustration of the MSW crossing. The dashed lines correspond to the electron-electron and muon-muon diagonal elements of the $m_{\nu}^{2}$ matrix in the flavor basis. Their intersection defines the level-crossing density $\rho_{c}$. The solid lines are the trajectories of the light and heavy local mass eigenstates. If the electron neutrino is produced at high density and propagates adiabatically, it will follow the heavy-mass trajectory, emerging from the sun as a $\nu_{\mu}$.

where $\theta_{i}=\theta\left(x_{i}\right)$ is the local mixing angle at the density where the neutrino was produced. (So if $\theta_{v} \sim 0$ and if the starting solar core density is sufficiently high so that $\theta_{i} \sim \pi / 2, p_{\nu_{e}}^{\text {adiab }} \sim 0$.)

The physical picture behind this derivation is illustrated in Figure 9. One makes the usual assumption that, in vacuum, the $\nu_{e}$ is almost identical to the light mass eigenstate, $\nu_{L}(0)$, i.e., $m_{1}<m_{2}$ and $\cos \theta_{v} \sim 1$. But as the density increases, the matter effects make the $\nu_{e}$ heavier than the $\nu_{\mu}$, with $\nu_{e} \rightarrow \nu_{H}(x)$ as $\rho(x)$ becomes large. The special property of the sun is that it produces $\nu_{e}$ 's at high density that then propagate to the vacuum where they are measured. The adiabatic approximation tells us that if initially $\nu_{e} \sim \nu_{H}(x)$, the neutrino will remain on the heavy mass trajectory provided the density changes slowly. That is, if the solar density gradient is sufficiently gentle, the neutrino will 
emerge from the sun as the heavy vacuum eigenstate, $\sim \nu_{\mu}$. This guarantees nearly complete conversion of $\nu_{e}$ 's into $\nu_{\mu}$ 's, producing a flux that cannot be detected by the Homestake or SAGE/GALLEX detectors.

Although it goes beyond the scope of this discussion, the case where the crossing is nonadiabatic can also be handled in an elegant fashion by following a procedure introduced by Landau and Zener for similar atomic physics level-crossing problems. The result [39] is an oscillation probability valid for all $\delta m^{2} / E$ and $\theta_{v}$

$$
p_{\nu_{e}}=\frac{1}{2}+\frac{1}{2} \cos 2 \theta_{v} \cos 2 \theta_{i}\left(1-2 e^{-\pi \gamma_{c} / 2}\right)
$$

As it must by our construction, $p_{\nu_{e}}$ reduces to $p_{\nu_{e}}^{\text {adiab }}$ for $\gamma_{c} \gg 1$. When the crossing becomes nonadiabatic (e.g., $\left.\gamma_{c} \ll 1\right)$, the neutrino "hops" to the light mass trajectory as it reaches the crossing point, allowing the neutrino to exit the sun as a $\nu_{e}$, i.e., no conversion occurs.

Thus there are two conditions for strong conversion of solar neutrinos: there must be a level crossing (that is, the solar core density must be sufficient to render $\nu_{e} \sim \nu_{H}\left(x_{i}\right)$ when it is first produced) and the crossing must be adiabatic. The first condition requires that $\delta m^{2} / E$ not be too large, and the second $\gamma_{c} \gtrsim 1$. The combination of these two constraints, illustrated in Figure 10, defines a triangle of interesting parameters in the $\frac{\delta m^{2}}{E}-\sin ^{2} 2 \theta_{v}$ plane, as Mikheyev and Smirnov found by numerical integration. A remarkable feature of this triangle is that strong $\nu_{e} \rightarrow \nu_{\mu}$ conversion can occur for very small mixing angles $\left(\sin ^{2} 2 \theta \sim 10^{-3}\right)$, unlike the vacuum case.

One can envision superimposing on Figure 10 the spectrum of solar neutrinos, plotted as a function of $\frac{\delta m^{2}}{E}$ for some choice of $\delta m^{2}$. Since Davis sees some solar neutrinos, the solutions must correspond to the boundaries of the triangle in Figure 10. The horizontal boundary indicates the maximum $\frac{\delta m^{2}}{E}$ for which the sun's central density is sufficient to cause a level crossing. If a spectrum properly straddles this boundary, we obtain a result consistent with the Homestake experiment in which low energy neutrinos (large 1/E) lie above the level-crossing boundary (and thus remain $\nu_{e}$ 's), but the highenergy neutrinos (small 1/E) fall within the unshaded region where strong conversion takes place. Thus such a solution would mimic nonstandard solar models in that only the ${ }^{8} \mathrm{~B}$ neutrino flux would be strongly suppressed. The diagonal boundary separates the adiabatic and nonadiabatic regions. If the spectrum straddles this boundary, we obtain a second solution in which low 


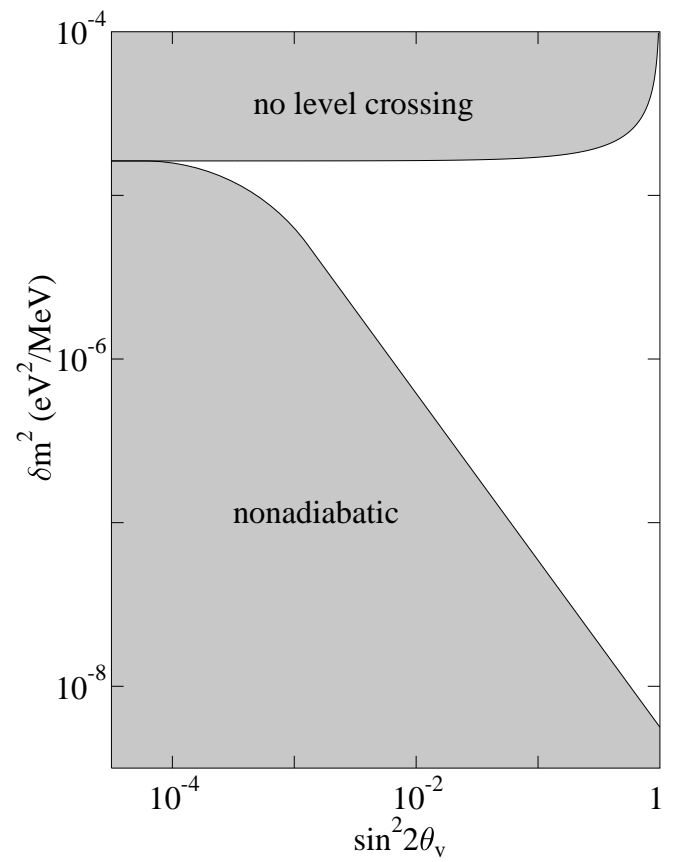

Figure 10: MSW conversion for a neutrino produced at the sun's center. The upper shaded region indices thoses $\delta m^{2} / E$ where the vacuum mass splitting is too great to be overcome by the solar density. Thus no level crossing occurs. The lower shaded region defines the region where the level crossing is nonadiabatic ( $\gamma_{c}$ less than unity). The unshaded region corresponds to adiabatic level crossings where strong $\nu_{e} \rightarrow \nu_{\mu}$ will occur.

energy neutrinos lie within the conversion region, but the high-energy neutrinos (small 1/E) lie below the conversion region and are characterized by $\gamma \ll 1$ at the crossing density. (Of course, the boundary is not a sharp one, but is characterized by the Landau-Zener exponential). Such a nonadiabatic solution is quite distinctive as the flux of pp neutrinos, which is strongly constrained in the standard solar model and in any steady-state nonstandard model by the solar luminosity, would now be sharply reduced. Finally, one can imagine "hybrid" solutions where the spectrum straddles both the levelcrossing (horizontal) boundary and the adiabaticity (diagonal) boundary for small $\theta$, thereby reducing the ${ }^{7}$ Be neutrino flux more than either the pp or 
${ }^{8} \mathrm{~B}$ fluxes.

Remarkably, this last possibility seems quite consistent with the experiments we have discussed. In fact, a nearly perfect fit to the data results from choosing $\delta m^{2} \sim 5 \cdot 10^{-6} \mathrm{eV}^{2}$ and $\sin ^{2} 2 \theta_{v} \sim 0.006$. As the $\delta m^{2}$ is quite different from that found in the atmospheric neutrino results, the mixing seen in solar neutrinos is distinct from that seen in atmospheric neutrinos, consistent with our attribution of the former to $\nu_{e} \rightarrow \nu_{\mu}$ oscillations and the latter to $\nu_{\mu} \rightarrow \nu_{\tau}$.

The argument that the solar neutrino problem must be due to neutrino oscillations is quite strong, but not as compelling as in the case of atmospheric neutrinos. Our conclusions follow from combining the results of several experiments, and not from direct observation of new physics, such as the zenith angle dependence in the atmospheric results. For this reason there is great interest in a new experiment now being readied in the Creighton nickel mine

in Sudbury, Ontario, 6800 feet below the surface. The Sudbury Neutrino Observatory (SNO) 40 has a central acrylic vessel filled with one kiloton of very pure $(99.92 \%)$ heavy water, surrounded by a shield of 7.5 kilotons of ordinary water. SNO can detect neutrinos through the charged current reaction

$$
\nu_{e}+d \rightarrow p+p+e^{-}
$$

as well as through the neutral current reaction

$$
\nu_{x}\left(\bar{\nu}_{x}\right)+d \rightarrow \nu_{x}\left(\bar{\nu}_{x}\right)+p+n
$$

Thus SNO offers the exciting possibility of comparing the solar flux in $\nu_{e}$ 's with that in all flavors, thereby providing a definitive test of flavor oscillations. The electrons from the first reaction above will be detected by the Cerenkov light they generate: SNO's central vessel is surrounded by 9800 phototubes. The neutrons produced in the neutral current reaction can be detected using either the $(n, \gamma)$ reaction on salt dissolved in the heavy water or proportional counters exploiting the ${ }^{3} \mathrm{He}(\mathrm{n}, \mathrm{p})^{3} \mathrm{H}$ reaction. SNO should begin taking data in summer, 1999.

\section{Conclusions}

In this article we have summarized some of the basic ideas of neutrino physics. Neutrinos come in three species or flavors $-\nu_{e}, \nu_{\mu}, \nu_{\tau}-$ with their correspond- 
ing antiparticles. We have discussed the unresolved problem of the nature of these antiparticles: are $\nu$ 's and $\bar{\nu}$ 's distinquished by some additive quantum number (Dirac neutrinos), or do they instead correspond to the two projections of opposite handedness of the same state (Majorana neutrinos)? We reviewed direct mass measurements, which have not yet yielded evidence for nonzero masses, and the reasons that the minimal standard model cannot accommodate massive neutrinos. Yet we have seen strong though indirect evidence for massive neutrinos in three classes of neutrino oscillation experiments. In an accelerator experiment - LSND - an unexpected flux of electron antineutrinos has been attributed to $\bar{\nu}_{\mu} \rightarrow \bar{\nu}_{e}$ oscillations. Over the past 15 years a series of experiments have been carried out on the neutrinos produced when high energy cosmic rays interact in the upper atmosphere. The increasing evidence of an anomaly has culminated with the SuperKamiokande measurements that confirm a deficit in the muon neutrino flux, and find a zenith angle dependence directly indicating oscillations. When constraints from reactor and accelerator experiments are taken into account, the explanation for the atmospheric neutrino anomaly appears to be $\nu_{\mu} \rightarrow \nu_{\tau}$ oscillations corresponding to a nearly maximal mixing angle. Finally, we discussed the evidence for a deficit in the flux of solar electron neutrinos and the difficulty in attributing this deficit to uncertainties in the solar model. Again the hypothesis of neutrino oscillations accounts for the observations, with one attractive possibility being a $\nu_{e} \rightarrow \nu_{\mu}$ oscillation enhanced by matter effects within the sun.

One popular choice for the mass differences and mixing angles needed to account for these observations is shown in Figure 11. The pattern that emerges is not, unfortunately, compatible with the simplest scenario of three mixed neutrinos: three distinct $\delta m^{2}$ values are required, while a threeneutrino scenario provides only two. This could mean that one (or more) of the experiments we have discussed has been misinterpretted. Alternatively, it could indicate the existence of additional light neutrinos that have so far avoided detection because they are sterile, lacking the usual standard model interactions. In any case, it is clear that the possibilities summarized in Figure 11 will require much more study in a new generation of heroic neutrino experiments. We have noted two efforts that made provide important results in the not too distant future: SNO will soon tell us whether there exists a $\nu_{\mu}$ or $\nu_{\tau}$ component in the solar neutrino flux, while a new Fermilab experiment will test the LSND conclusions. Thus perhaps the picture will 


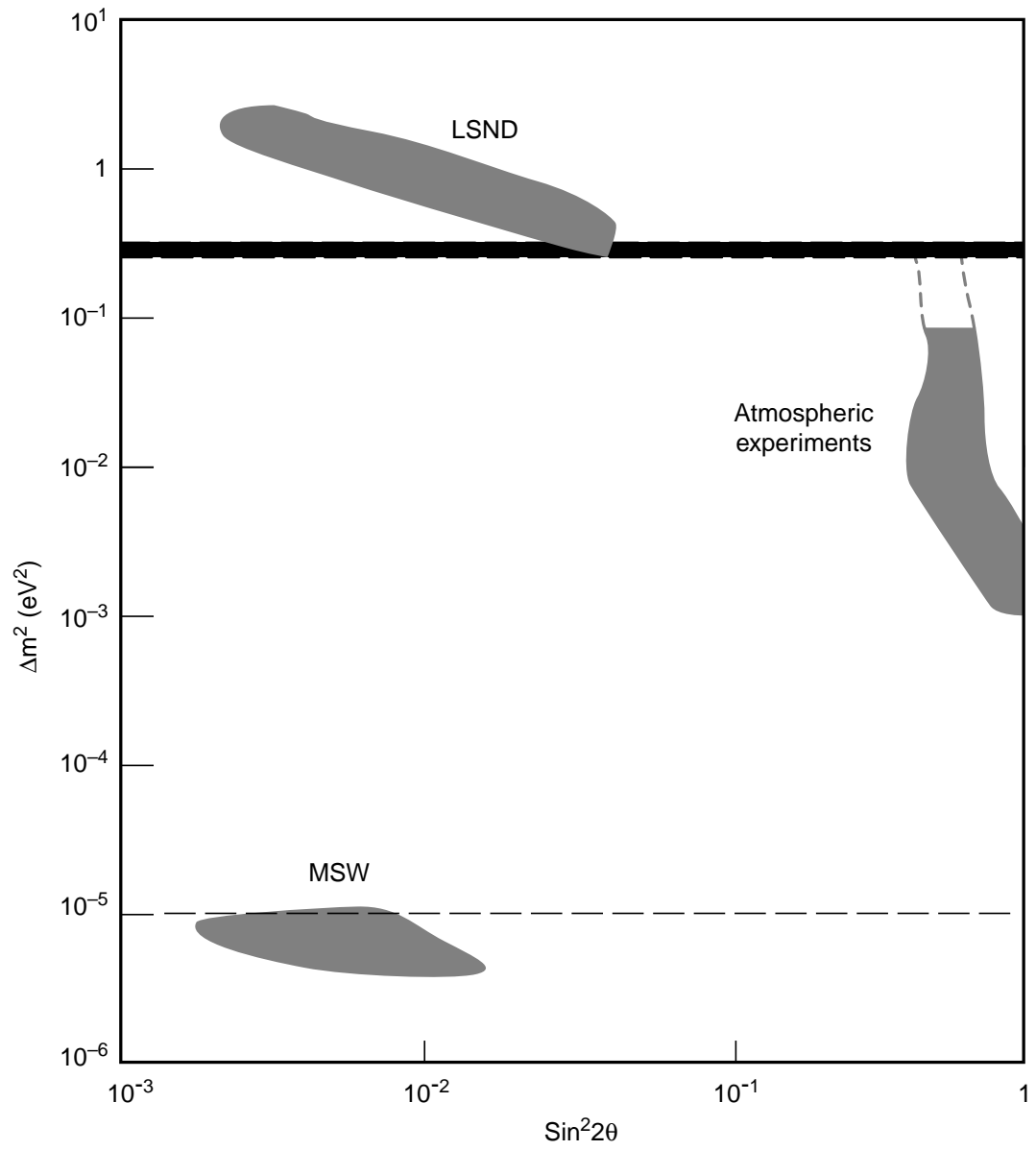

Figure 11: One set of $\delta m^{2}$ and $\sin ^{2} 2 \theta$ regions that could account for the solar neutrino, atmospheric neutrino, and LSND results. This figure was provided by Vern Sandberg. 
become far clearer in the next few years. In the meantime, neutrinos will remain in the news. We have tried to illustrate how the many profound issues connected with neutrinos can be readily appreciated with only simple quantum mechanics. It is our hope, therefore, that the developing discoveries in neutrino physics - which may show the way to the next standard model of particle physics - will be accessible to and followed by beginning students of physics. We hope the material presented here, which is suitable for beginning courses in quantum mechanics and modern physics, will help make this possible.

\section{Acknowledgement}

This work is supported in part by the National Science Foundation (BRH) and by the Department of Energy (WCH). In addition BRH would like to acknowledge the support of the Alexander von Humboldt Foundation and the hospitality of Forschungszentrum Jülich. We also thank Bill Louis and Vern Sandberg for providing Figures 7 and 11.

\section{References}

[1] See, e.g. "A Massive Discovery," in Sci. Am., August (1998), pp. 18-20.

[2] J. Updike, From Telephone Poles and Other Poems, A. Knopf, New York (1963).

[3] L.M. Brown, "The Idea of the Neutrino," Physics Today, September 1978, 23-28; also see "Celebrating the Neutrino," Los Alamos Science 25, 1-191 (1997).

[4] A translation of Pauli's contribution ("Aufsätze and Vorträge über Physik and Erkenntnistheorie," Proc. VII Sovay Congress, Brussels (1933), p. 324) can be found in ref. [3].

[5] E. Fermi, "Versuch einer Theorie der $\beta$-Strahlen," Z. Phys. 88, 161-77 (1934).

[6] C.L. Cowan, Jr., F. Reines, F.B. Harrison, E.C. Anderson, and F.N. Hayes, "Detection of the Free Neutrino: a Confirmation," Science 124, 103-04 (1956). 
[7] G. Danby, J.-M. Gaillard, K. Goulianos, L.M. Lederman, N. Mistry, M. Schwartz, and J. Steinberger, "Observation of High-Energy Neutrino Reactions and the Existence of Two Kinds of Neutrinos," Phys. Rev. Lett. 9, 36-44 (1962).

[8] Often quoted is Rabi's remark about the discovery of the muon- "Who ordered that?"

[9] Of course, decay of the $\pi^{+}$to an electron and neutrino is also possible but is highly suppressed for helicity reasons, as we shall discuss below.

[10] See, e.g. J.F. Donoghue, E. Golowich, and B.R. Holstein, Dynamics of the Standard Model, Cambridge Univ. Press, New York (1992), Ch. XVI-2

[11] G. Steigman, D. Schramm, and J. Gunn, "Cosmological Limits to the Number of Massive Leptons," Phys. Lett. B66, 202-04 (1978); J. Yang, et al., "Primeval Nucleosynthesis: a Critical Comparison of Theory and Observation," Ap. J. 281, 493-511 (1984).

[12] J. D. Bjorken and S. D. Drell, Relativistic Quantum Mechanics, McGraw-Hill, New York (1965).

[13] In priniciple there could be right-handed neutrinos present, but they could not interact and hence we would not be aware of their presence.

[14] M. Goldhaber, L Grodzins, and A. Sunyar, "Helicity of Neutrinos," Phys. Rev. 109, 1015-17 (1958).

[15] Particle Data Group, Phys. Rev. D54, 321 (1996).

[16] W. C. Haxton and G. J. Stephenson, Jr., "Double Beta Decay," Prog. Part. Nucl. Phys. 12, 409-479 (1984).

[17] See, for example, talks by H. V. Klapdor-Kleingrothaus and A. Morales, Neutrino '98 (Takayama, Japan) June, 1998.

[18] C.S. Wu, "The Experimental Determination of the Beta-Interaction(B) the Rest Mass of the Electron," in Alpha- Beta- and GammaRay Spectroscopy, ed. K. Siegbahn, North-Holland, Amsterdam (1965), Vol. 2, pp. 1391-96. 
[19] K.-E. Bergkvist, "A High-luminosity, High Resolution Study of the Endpoint Behavior of the Tritium $\beta$-spectrum," Nucl. Phys. B39, 317-70 (1972).

[20] V.A. Lubimov, E.G. Novikov, V.Z. Nozik, E.F. Tretyakov, and V.S. Kosik, "An estimate of the $\nu_{e}$ Mass from the $\beta$-spectrum of Tritium in the Valine Molecule," Phys. Lett. B94, 266-68 (1980).

[21] The constant in front of the neutrino expression differs from the corresponding photon number because of the use of Fermi rather than Bose statistics

$$
\int_{0}^{\infty} d z \frac{z^{3}}{e^{z}-1}=\frac{\pi^{4}}{15} \rightarrow \int_{0}^{\infty} d z \frac{z^{3}}{e^{z}+1}=\frac{7 \pi^{4}}{120}
$$

[22] R.G.H. Robertson et al., "Limit on Electron Antineutrino Mass from Observation of the $\beta$-Decay of Molecular Tritium," Phys. Rev. Lett. 67, 957-60 (1991).

[23] Ch. Weinheimer et al., "Improved Limit on the Electron-Antineutrino Rest Mass for Tritium $\beta$-Decay," Phys. Lett. B300, 210-16 (1993).

[24] W. Stoeffl and D.J. Decman, "Anomalous Structure of the Beta Decay of Gaseous Molecular Tritium," Phys. Rev. Lett. 75, 3237-40 (1995).

[25] S. Chandrasekhar, "The Highly Collapsed Configurations of Stellar Mass," Mon. Not. Roy. Ast. Soc. 95, 207-25 (1935); L. D. Landau, "On the Theory of Stars," in Collected Papers of L. D. Landau, ed D. ter Haar, Gordon and Breach, New York (1965), pp.60-62, originally published in Phys. Z. Sowjet Union 1, 285 (1932).

[26] B. Pontecorvo, "Neutrino Experiments and the Problem of Conservation of Leptonic Charge," Sov. Phys. JETP 26, 984-88 (1968).

[27] The $\bar{\nu}_{e}$ 's come from both the fraction of $\mu^{-}$'s that decay via $\mu^{-} \rightarrow$ $e^{-}+\bar{\nu}_{e}+\nu_{\mu}$ and from the small percentage of negatively charged pions that decay via $\pi^{-} \rightarrow e^{-}+\bar{\nu}_{e}$.

[28] C. Athanassopoulos et al., "Candidate Events in a Search for $\bar{\nu}_{\mu} \rightarrow \bar{\nu}_{e}$ Oscillations," Phys. Rev. Letters 75, 2650-53 (1995) and "Evidence for $\bar{\nu}_{\mu} \rightarrow \bar{\nu}_{e}$ Oscillations from the LSND Experiment at the Los Alamos Meson Physics Facility," Phys. Rev. Lett. 77, 3082-85 (1996). 
[29] Y. Fukuda et al., "Measurement of a Small Atmospheric $\nu_{\mu} / \nu_{e}$ Ratio," Phys. Rev. Lett. 81, 1562-5 (1998).

[30] M. Gell-Mann, P. Ramond, and R. Slansky in Supergravity, ed. P. van Hiewenhuizen and D. Z. Freedman, North Holland, Amsterdam, 1979, p 315; T. Tanagida, in Proceedings of the Workshop on the Unified Theory and Baryon Number in the Universe, ed. O. Sawada and A. Sugamoto, p. 95.

[31] F. Wilczek, talk presented at Neutrino '98 (Takayama, Japan) June, 1998.

[32] J. N. Bahcall, S. Basu, and M. H. Pinsonneault, "How Uncertain are Solar Model Predictions?" Phys. Letts. B 433, 1-8 (1998); A. S. Brun, S. Turck-Chieze, and P. Morel, "Standard Solar Models in the Light of Helioseismic Constraints: I. The Solar Core," Ap. J. 506, 913-925 (1998).

[33] R. Davis, Jr., D.S. Harmer, and K.C. Hoffman, "Search for Neutrinos from the Sun," Phys. Rev. Lett. 20, 1205-12 (1968); R. Davis, Jr., "A Review of the Homestake Solar Neutrino Experiment," Prog. Part. Nucl. Phys. 32, 13-32 (1994).

[34] Y. Fukuda et al., "Solar Neutrino Data Covering Solar Cycle 22," Phys. Rev. Lett. 77, 1683-86 (1996).

[35] R. Anselmann et al., "GALLEX Results from the First 30 Solar Neutrino Runs," Phys. Lett. B327, 377-85 (1994) and "First Results from the ${ }^{51}$ Cr Neutrino Source Experiment with the GALLEX Detector," Phys. Lett. B342, 440-50 (1995); J.N. Abdurashitov et al., "Results from SAGE," Phys. Lett. B328, 234-48 (1994).

[36] S.P. Mikheyev and A. Yu. Smirnov, "Resonance Enhancement of Oscillations in Matter and Solar Neutrino Spectroscopy," Sov. J. Nucl. Phys. 42, 913-17 (1985) and "Resonant Amplification of $\nu$ Oscillation in Matter and Solar Neutrino Spectroscopy," Nuovo Cimento 9C, 17-26 (1986).

[37] L Wolfenstein, "Neutrino Oscillations in Matter," Phys. Rev. D17, 2369-74 (1978). 
[38] H. Bethe, "Possible Explanation of the Solar-Neutrino Puzzle," Phys. Rev. Lett. 56, 1305-8 (1986).

[39] W. C. Haxton, "Adiabatic Conversion of Solar Neutrinos," Phys. Rev. Lett. 57, 1271-4 (1986); S. J. Parke, "Nonadiabatic Level Crossing in Resonant Neutrino Oscillations," Phys. Rev. Lett. 57, 1275-8 (1986).

[40] G.T. Ewan et al., "The Building of the Sudbury Neutrino Observatory," Physics in Canada 48 (2) (1992). 